\title{
NBSIR $82-2457$
}

\section{Gram Capacity Combustion Flow Calorimeter}

U.S. DEPARTMENT OF COMMERCE

National Bureau of Standards

Chemical Thermodynamics Division and the

Temperature Pressure Measurements and Standards Division

Washington, DC 20234

March 1982

Prepared for

U.S. Department of Energy

Office of Energy from Municipal Waste

- QC - $\quad$ - $\quad 00$ Independence Avenue, SW

100 shington, DC 20585

.456

ن2-2457

1962

C. 2 



\section{GRAM CAPACITY COMBUSTION} FLOW CALORIMETER

E.S. Domalski, K.L. Churney, M.L. Reilly, D.R. Kirklin, A.E. Ledford and D.D. Thornton

U.S. DEPARTMENT OF COMMERCE

National Bureau of Standards

Chemical Thermodynamics Division and the

Temperature Pressure Measurements and Standards Division

Washington, DC 20234

March 1982

Prepared for

U.S. Department of Energy

Office of Energy from Municipal Waste

Forrestal Building

9000 Independence Avenue, SW

Washington, DC 20585

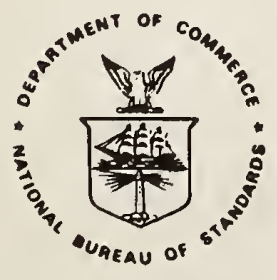

U.S. DEPARTMENT OF COMMERCE, Malcolm Baldrige, Secretary NATIONAL BUREAU OF STANDARDS, Ernest Ambler, Director 
\% in in th th . . ?

$\therefore i+y^{2}$ 


\section{Table of Contents}

Page

1. Introduction .................. 1

2. 25 Gram Flow Calorimeter Experimental Work . . . . . . . 4

2.1 Apparatus and Samples ........... 4

2.2 Description of a Typical Experiment . . . . . 8

3. Calculation of 25 Gram Flow Results .......... 10

3.1 Calculation of $\Delta T \ldots \ldots$.............. 11

3.2 Calculation of Enthalpy Changes. . . . . . . 14

3.3 Calculation of $\Delta_{\mathrm{st}}$ from Bomb Calorimetry. . . . 15

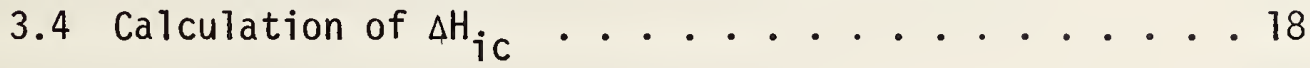

3.5 Calculation of $\Delta H_{g} \ldots \ldots 21$

3.6 Calculation of $\Delta H_{v} \ldots \ldots . . . . . . .22$

3.7 Calculation of $\Delta H_{i g n} \ldots \ldots . . . . . . .26$

3.8 Summary of Calculations. . . . . . . . . 26

4. Systematic Error Analys is of 25 Gram Flow Experiments . . . . 26

4.1 Errors in $\Delta R \ldots . \ldots . \ldots 29$

4.2 Errors in $\Delta H_{s t} \ldots \ldots 30$

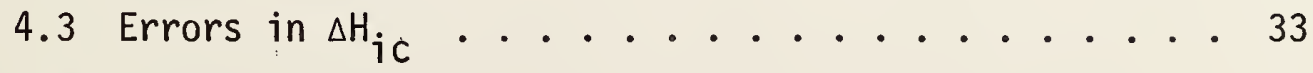

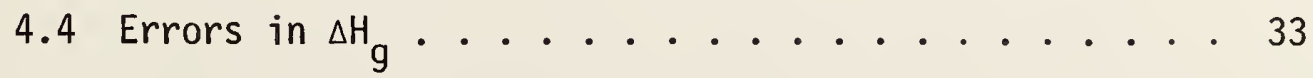

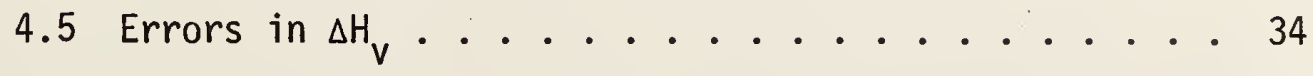

4.6 Summary of Error Analysis . . . . . . . . . . 34

5. Results of 25 Gram Flow Experiments and Conclusions . . . . 36

6. References .................. 42

7. Acknowledgements ............... 43 

1. Flow Calorimetric Measurements on RDF-4 samples;

Calculation of the Corrected Temperature Rise . . . . . . 13

2. Bomb Calorimetric Measurements on RDF-4 Samples . . . . . 16, 17

3. Standard Flow Reaction ............... 19

4. Conversion of Calorimetric Data from Bomb to Flow Conditions ....................... 20

5. Calculation of Moles of Water Vaporized from the Calorimeter. ...................... 25

6. Sample Mass and Molar Quantities for Flow Calculations ... 27

7. Calculation of 25 Gram Flow Calorimeter Results . . . . . 28

8. Acid Content of Water Trap .............. 31

9. Systematic Error Analysis of 25 Gram Flow Experiments . . . 35

10. Summary of 25 Gram Flow Results . . . . . . . . . 37

11. Comparison of Flow and Bomb Calorimeter Results ...... 38 


\section{List of Figures}

Page

Figure 1

The 25 Gram Combustor............ . .

Figure 2

The 25 Gram Flow Calorimeter

6

Figure 3

The $\mathrm{CO}-\mathrm{CO}_{2}$ Composition of the Product Gases vs.

Elapsed Time from Ignition for a Typical

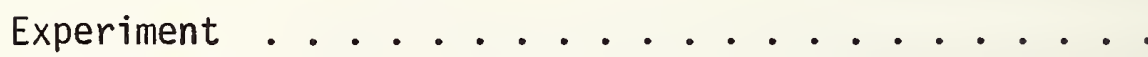




\section{GRAM CAPACITY COMBUSTION FLOW CALORIMETER}

E.S. Domalski, K.L. Churney, M.L. Reilly, D.R. Kirklin,

A. E. Ledford, and D. D. Thornton

\section{ABSTRACT}

A new calorimeter is being developed at the National Bureau of Standards to determine the enthalpies of combustion of kilogram-size samples of municipal solid waste (MSW) in flowing oxygen near atmospheric pressure. The organic fraction of 25 gram pellets of highly processed MSW has been burned in pure oxygen to $\mathrm{CO}_{2}$ and $\mathrm{H}_{2} \mathrm{O}$ in a small prototype flow calorimeter. The carbon content of the ash and the uncertainty in the amount of $\mathrm{CO}$ in the combustion products contribute calorimetric errors of 0.1 percent or less to the enthalpy of combustion.

\section{Introduction}

The National Bureau of Standards (NBS) has been mandated by the U.S. Congress through Public Law 94-580, the Resource Conservation and Recovery Act of 1976, to develop guidelines for specifications on recoverable waste materials. NBS has made a commitment to develop a calorineter to determine the calorific value of kilogram-size samples of minimally processed municipal solid waste (MSW). A large scale calorimeter affords greater credibility because kilogram-size samples of MSW should be more representative of the heterogeneous bulk material than the highly processed gram-size samples currently used in bomb calorimetric determinations.

Combustion of samples in flowing oxygen near atmospheric pressure rather than in the high pressure oxygen of a combustion bomb was adopted for safety considerations. However, the flow technique has not been used in any substantial way to determine the enthalpy of combustion of solids since the $1880^{\prime} s$. Its development was dis- 
continued because of the simpler techniques and more quantitative results obtained with the bomb calorimeter. Attaining complete combustion was particularly difficult in a flow system. As a consequence, the first goal of the NBS project was to demonstrate that the oxygen flow technique could be used to obtain complete combustion of MSW.

The first step in the program was to build a calorimeter for combustion of 25 gram pellets of highly processed MSW. The sample was burned in the form of pellets to simplify ash collection. The purpose was to establish the equivalence of the flow results with those obtained by bomb calorimetry (e.g. with the new NBS 25 gram capacity bomb calorimeter [1]). The preliminary results obtained with the 25 gram flow calorimetric system are described in the following sections.

The early development of the combustor for the 25 gram capacity flow calorimeter dates back to December 1978. An acceptable design and suitable operating procedures for this combustor were established as a result of combustion studies completed in the sumner of 1979. Calorimetric measurements were conducted and analyzed between October 1979 and July 1980. The prototype flow calorimeter was used for these measurements while the final calorimeter was being fabricated. All measurements described below were made with the prototype calorimeter. Measurements with the final calorimeter will be started in fiscal year 1981.

Benzoic acid was found to be unsatisfactory as a solid calibrant material for flow work. Pellets melted and burned with a flame that produced large amounts of soot. Ultra-pure carbon powder also proved to be unsatisfactory; it was very difficult to ignite. Flame calorimetry calibrants (gaseous $\mathrm{H}_{2}$ or $\mathrm{CH}_{4}$ ) were not appropriate because an apparatus for quantitative (i.e. $0.01 \%$ ) determination of the amounts of calibrant combusted had not been constructed.

The prototype flow calorimeter was calibrated by burning five pellets of RDF-4 from Lot $A$, whose enthalpy of combustion had been determined previously with 
a conventional gram-size bomb calorimeter at NBS. The heat of combustion of a different RDF-4, called Lot B, was measured in six experiments. The ash was determined for each experiment in order to verify that the combustible fraction of each lot was the same within experimental uncertainty for both bomb and flow measurements.

After the RDF-4 experiments, one gram pellets of pure cellulose were test burned in a similar combustor. The pellets ignited easily and burned completely. Subsequently, bomb calorimetric measurements were initiated to establish the enthalpy of combustion of this material so that it can be used as a standard solid calibrant for the flow calorimetric measurements.

The experimental measurements on Lot $A$ and Lot $B$ are described in section two and the calculation of results and an error analysis of the results are given in sections three and four. Results and conclusions are given in section five. 


\section{25 Gram Flow Calorimeter Experimental Work}

\subsection{Apparatus and Samples}

The combustor used in the 25 gram oxygen flow experiments is shown in Fig. 1. The sample pellet (D of Fig. 1) was placed on a quartz plate (F) which had eight radial slots and a small central hole which permitted circulation of oxygen beneath the sample. The plate sat in a quartz crucible (E) which was supported by a nichrome stand $(J)$. Primary oxygen $(H)$ was supplied locally to the sample through a three-port tubular quartz ring which was located immediately above the crucible. The lower edge of the Pyrex thermal shield (C) enclosed the ring and upper edge of the crucible. The outer boundary of the combustor consisted of the stainless steel top (N) and base (I) plus the Pyrex wall (L) which were sealed by rubber gaskets $(B)$. A secondary flow of oxygen $(G)$ swept down between the wall of the combustor and the thermal shield and confined the flow of the products of combustion to the interior of the thermal shield. Gases left the combustor through a stainless steel exit line (A) in the top. The temperatures of the product gases near the exit port of the thermal shield and of the midpoint of the interior of the thermal shield were monitored using Type $K$ thermocouples. The thermocouples were supported in helical Pyrex tubing $(K)$ which stood within the thermal shield.

The combustor was placed in a provisional calorimetric assembly similar to the final design shown in Fig. 2. The provisional assembly consisted of an insulated bell jar filled with water which was circulated by a stirrer ( $J$ of Fig. 2) mounted from the bell jar cover. The flow shield (E) caused the water to circulate up past the combustor ( $L$ ) and down along the inner wall of the jar (analogous to $B$ ). The thin metal disk $(K)$ mounted on the stirrer shaft eliminated leakage of water through the Teflon seal between the stirrer shaft and the bell jar cover (cf. between the shaft and A of Fig. 2). The temperature of the water was measured 


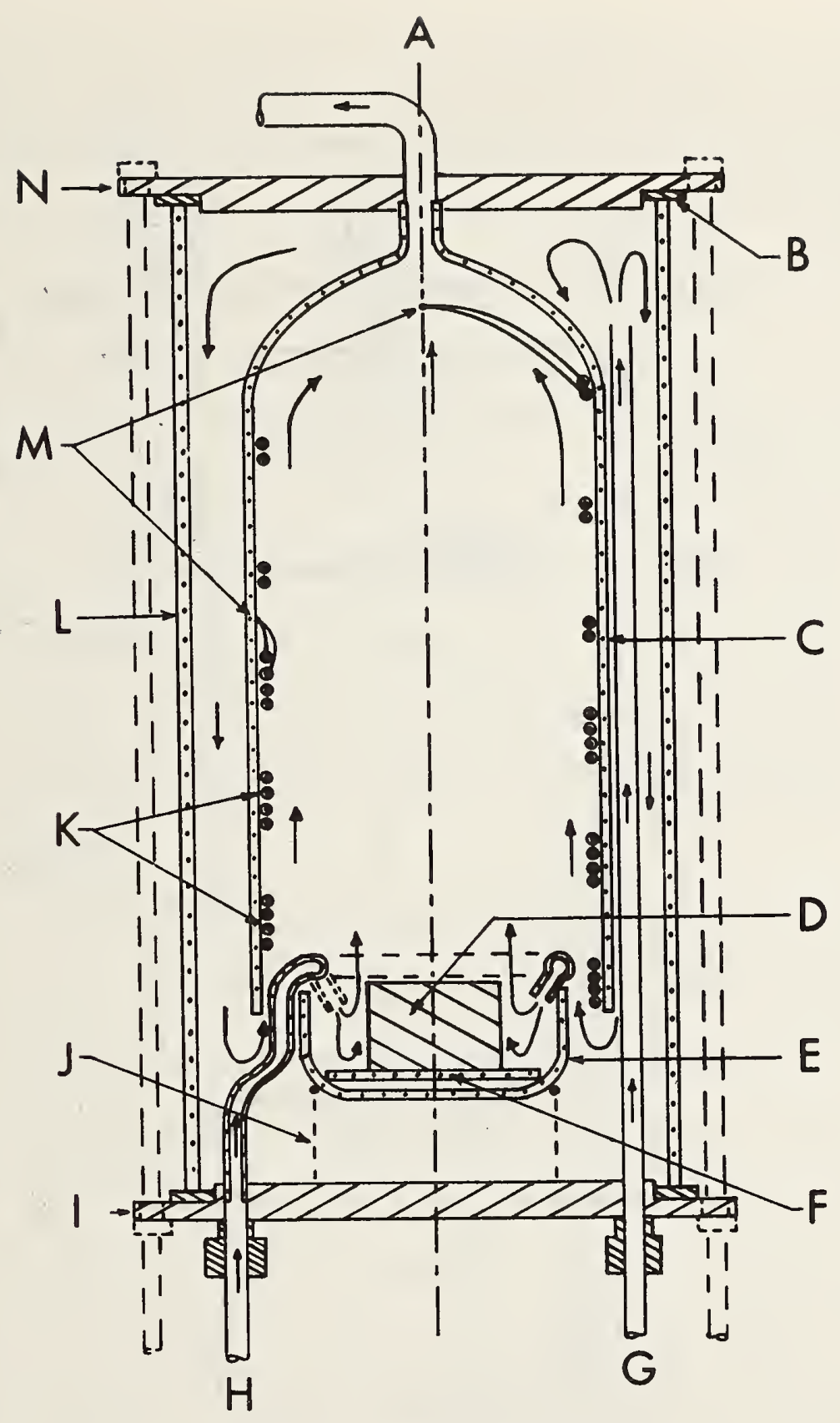

$16 \mathrm{~cm} \longrightarrow$

Fig. 1. The 25 Grain Combustor.

Notations: A denotes the product gas exit line, $B$ the gasket, $C$ the thermal shield, D the RDF-4 sample, E the crucible, $\mathrm{F}$ the plate, $\mathrm{G}$ the secondary oxygen, $\mathrm{H}$ the primary oxygen, I the combustor base, $J$ the crucible support, $K$ the thermocouple coils, $L$ the combustor walls, $M$ the thermocouple junctions and $\mathrm{N}$ the combustor lid. 


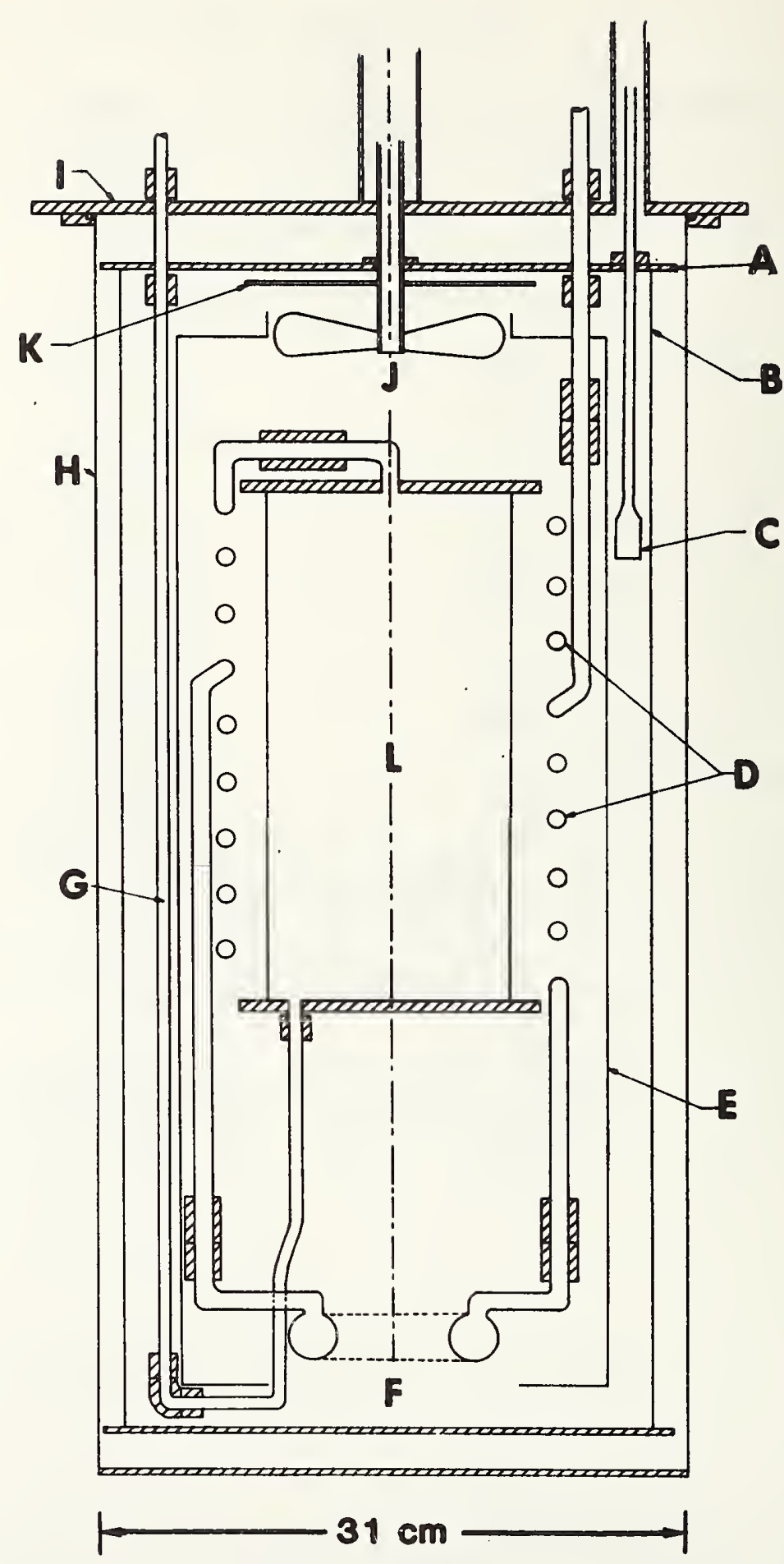

Fig. 2. The 25 Gram Flow Calorimeter.

Notations: A denotes the vessel iid, B the vessel can, C the thermometer, D the exit gas coils, E the flow shield, $F$ the water trap, $G$ the oxygen supply lines, $\mathrm{H}$ the submarine can, I the submarine lid, J the stirrer, $K$ the metal disk and $L$ the combustor. 
with a long stem platinum resistance thermometer $(C)$. The product gases flowed through the helical exit gas coil (D) into the water trap (F) and then through a second coil before leaving the calorimeter. The coils and trap were made from Pyrex.

After leaving the calorimeter, the product gases were scrubbed by bubbling through water and then were diluted with dry gas before the entire gas stream was passed successively through a variable orifice flowmeter and non-dispersive infrared detectors for measuring the concentrations of $\mathrm{CO}$ and $\mathrm{CO}_{2}$. Analysis of the product gases in the combustor development experiments by both mass spectrometry and conventional infrared spectroscopy showed $\mathrm{CO}$ to be the only species present due to incomplete combustion.

Ordinarily, the oxygen supplied to the calorimeter was saturated with water vapor by bubbling the gas through two gas washing flasks connected in series.

Type $K$ thermocouples were used to measure the temperature of the oxygen as it entered the calorimeter and the temperature of the product gases both as they left the calorimeter and at the $\mathrm{CO}$ and $\mathrm{CO}_{2}$ detectors. The pressure of the product gases at the $\mathrm{CO}$ and $\mathrm{CO}_{2}$ detectors was monitored with a capacitance digital manometer. The analog output of the $\mathrm{CO}, \mathrm{CO}_{2}$, and pressure detectors and all thermocouple voltages were monitored using a $51 / 2$ digit voltmeter ( $1 \mu V$ sensitivity), a ten channel scanner, and a 16-bit minicomputer. Calorimeter water temperatures were measured manually by determining the resistance of the platinum resistance thermometer with a Mueller bridge and an electronic null detector.

Sample pellets for the 25 gram experiments were prepared from a blended powder of minus $0.5 \mathrm{~mm}$ particle size. The powder was made by milling large batches of minus $2 \mathrm{~cm}$ MSW from which most of the metals, glass and entrained inorganics had been removed. This powder is referred to as RDF-4. The cylindrical pellets, which were $3.5 \mathrm{~cm}$ in diameter and a $2.5 \mathrm{~cm}$ high, were prepared by pressing the powder in a die using a force ranging from 45 to $160 \mathrm{kN}(10,000$ to $36,000 \mathrm{lbs})$. 
The residual moisture content of the powder was determined to be about $5 \%$ using the ASTM test E790, test for residual moisture in a refuse-derived fuel analysis sample.

\subsection{Description of a Typical Experiment}

In a typical experiment, the calorimeter was assembled and the combustor was flushed with pure oxygen. The primary and secondary oxygen flow rates were set at approximately 8 and 5 liters per minute $\left(L \cdot \mathrm{m}^{-1}\right)$, respectively. The temperature of the calorimeter water was recorded as a function of time for approximately twenty minutes after a steady drift rate was attained. The sample was ignited by passing electrical current through an iron fuse wire (not shown in Fig 1) which was in contact with the top of the pellet. A 25 gram pellet typically burned within fourteen minutes. Upon ignition the pellet burned with a diffusion flame which spread over its entire surface within the first 30 seconds. The flame temperature was determined to be greater than $1500{ }^{\circ} \mathrm{C}$. A small but detectable peak in the $\mathrm{C} 0$ concentration occurred at about 3 minutes. The surface flame disappeared at about the eighth minute. The glowing sample continued to burn internally leaving a porous ash structure that had almost the same shape as the original pellet. The product gases contained increasing amounts of $\mathrm{CO}$, which reached a peak near the eleventh minute. However, the ratio of $\mathrm{CO}$ to $\mathrm{CO}_{2}$ remained low. A typical $\mathrm{CO}-\mathrm{CO}_{2}$ composition profile of the product gases is shown in Fig 3 .

The temperature of the product gas stream at the top of the combustor rose quickly to a peak near $600{ }^{\circ} \mathrm{C}$ some 30 seconds after ignition and then decayed exponentially as the combustion proceeded. The product gases were cooled in the exit gas coils and approximately $85 \%$ of the water formed was collected in the trap. The gas was further cooled in the second exit coil. Throughout the combustion, the temperature of the product gases leaving the calorimeter was less than $0.1{ }^{\circ} \mathrm{C}$ above the temperature of the calorimeter water.

During the first seven minutes the temperature of the calorimeter water rose linearly at a rate near $0.25^{\circ} \mathrm{C} / \mathrm{min}$. Subsequently, the temperature of the calorimeter water continued to rise at a progessively diminishing rate until a final 


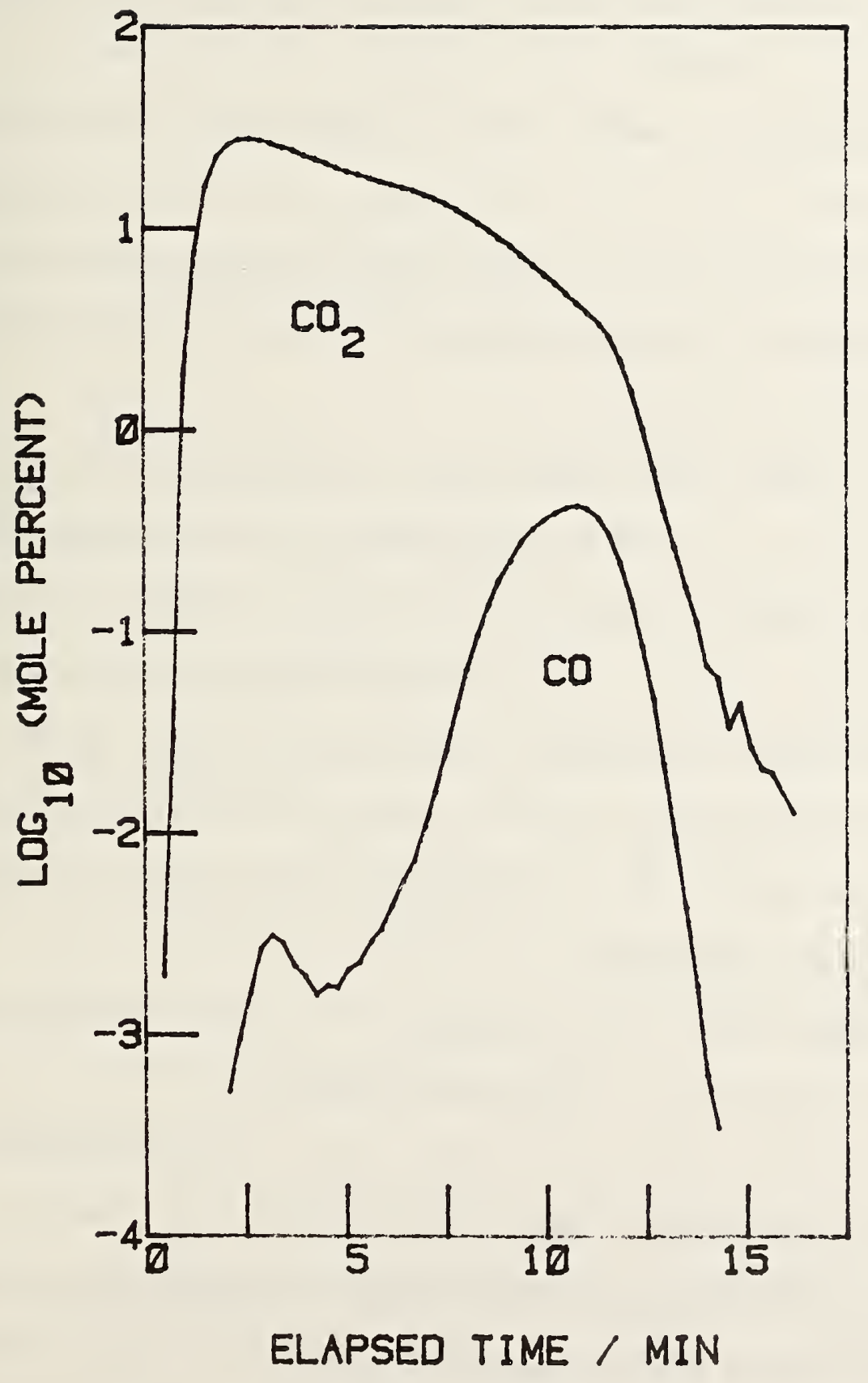

Fig. 3. The $\mathrm{CO}-\mathrm{CO}_{2}$ Composition of the Product Gases vs the Elapsed Time from Ignition for Typical Experiment. 
steady-state drift rate was attained about forty to forty-five minutes after ignition. To establish the final drift rate, temperatures were recorded for at least thirty additional minutes. The temperature rise (from the end of the initial drift period to the beginning of the final drift period) was typically $2.3{ }^{\circ} \mathrm{C}$.

After disassembly of the calorimeter, the ash and the sample crucible were weighed. The carbon content of the ash was determined from the sum of the mass decrease produced by heating the ash and crucible with an oxygen-gas flame plus subsequent analysis for the remaining total occluded carbon. The carbon in the ash ranged from 0.015 to $0.1 \%$ of the initial sample mass.

A small amount ( $<1 \mathrm{mg}$ ) of white residue was deposited on the thermal shield during each run. In about one-half of the experiments, the water condensed in the trap (see Fig. 2) was greenish blue rather than clear. The trap also contained a black residue. Apparently this was trapped fly ash which had partially dissolved. Analysis showed that no carbon was present in the residue and that the colored solution contained $\mathrm{Fe}, \mathrm{Cr}, \mathrm{Ni}, \mathrm{Na}$ and $\mathrm{K}$ (i.e. in the range of $100 \mu \mathrm{g} \cdot \mathrm{ml}^{-1}$ ) in addition to the usual acidity ( 0.1 to 0.2 milliequivalents per milliliter).

\section{Calculation of 25 Gram Flow Results}

Results are calculated using a format adapted from the work of Prosen et a1. [2] which treats only the case where all reactants are gaseous.

$$
E \Delta T / m=-\left(\Delta H_{s t}+\Delta H_{i c}+\Delta H_{g}+\Delta H_{v}+\Delta H_{i g n}\right)
$$

In eq. 1, $E$ is the energy equivalent of the calorimeter,

$\Delta T$ is the corrected temperature rise of the calorimeter,

$m$ is the mass of the pelleted RDF-4 sample,

$\Delta H_{s t}$ is the enthalpy change of an assumed standard flow reaction for one gram of RDF-4 in which all combustion products are at the same reference temperature $T_{h}$. 
The remaining four enthalpy change terms (per gram of RDF-4) on the right side of eq. 1 account for the conditions of a particular experiment. They are as follows:

$\Delta \mathrm{H}_{i \mathrm{C}}$ is the correction for the incomplete combustion of all $\mathrm{CO}$ to $\mathrm{CO}_{2}$, $\Delta \mathrm{H}_{\mathrm{g}}$ is the correction for the net heat transport by oxygen entering and product gases leaving the calorimeter at some temperature other than $T_{h}$, $\Delta \mathrm{H}_{v}$ is the correction for the heat of vaporization of water formed in the combustion that is lost from the calorimeter with the product gases,

and

$\Delta H_{i g n}$ is the correction for the enthalpy of combustion of the iron fuse wire used to ignite the RDF-4 sample.

The electrical work done to heat the fuse wire in order to ignite the sample has been assumed to be negligible.

An energy equivalent of the calorimeter was calculated for each of the experiments involving Lot $A$ using a value of $\Delta H_{s t}$ derived from bomb calorimetric measurements. The value of $\Delta H_{s t}$ for Lot $B$ was calculated using the average $E$ from the Lot $A$ experiments. The method used to calculate $\Delta T$ is given in section 3.1 and the enthalpy terms on the right side of eq. 1 are given in section 3.2 to 3.7. Results are tabulated in section 3.8.

\subsection{Calculation of $\Delta T$}

The value for $\Delta T$ is calculated in the same manner as in bomb calorimetry. The temperature of the calorimeter water was measured with the platinum thermometer immersed in the stirred water outside of the flow shield. As the resistance, $R$, of the thermometer varies nearly linearly with $T$ over the range of temperature $\left(<4{ }^{\circ} \mathrm{C}\right)$ involved in calculating $\Delta T$, the corrected resistance rise, $\Delta R$, was calculated in place of $\Delta T$, using eq. 2. The only effect is to change the units of $\mathrm{E}$ to $\mathrm{J} \cdot \Omega^{-1}$ (joules per ohm); $1 \Omega$ is approximately $10{ }^{\circ} \mathrm{C}$. 


$$
\Delta R=R_{f}-R_{i}-k \int_{t_{i}}^{t_{f}}\left(R_{\infty}-R\right) d t
$$

In eq. $2, R_{f}$ and $R_{i}$ are resistances at the end, time $t_{f}$, and the beginning, time $t_{j}$, of the main period. The symbols $k$ and $R_{\infty}$ are called the cooling constant and the convergence resistance, respectively. The values of $k$ and $R_{\infty}$ are calculated for each experiment by simultaneously fitting eq. 3 to both the initial and final drift data using a computer program written by R.L. Nuttali [3].

$$
d R / d t=k\left(R_{\infty}-R\right)
$$

The results are summarized in table 1. Experiment numbers in column 1 indicate the order in which they were performed followed by a letter indicating the lot designation.

Oxygen was supplied to the calorimeter at a constant flow rate throughout each experiment. In experiments 1 through 9 , the inlet oxygen was saturated with water vapor. The assumption was made that $k$ and $R_{\infty}$ were the same as if no oxygen was flowing during either drift period since this is, strictly speaking, required for the validity of eqs. 1 through 3 . The length of the main period was taken to be 41 minutes or longer, except for experiment $5 B$ where insufficient final drift data were available. Main periods of shorter duration yielded values of $k$ smaller than those in table 1 which indicate eq. 3 does not apply until $t_{f}-t_{j} \geq 41$ minutes.

The oxygen supplied to the calorimeter in experiments 10 and 11 was dry. Values of $k, R_{\infty}$ and $\Delta R$ in experiments 10 and 11 were adjusted to the average $k$ of the first nine experiments. It was assumed that the observed final drift rates in experiments 10 and 11 were too small due to vaporization of water from the calorimeter in the final drift period. The correction, $\delta \Delta R$, added to $\Delta R$ was:

$$
\delta \Delta R=-\left(k-k_{c}\right)\left[\left(R_{\infty}-R_{j}\right)\left(t_{f}-t_{j}\right)-\int_{t_{j}}^{\tau_{f}}\left(R_{\infty}-R\right) d t\right]
$$


O

离

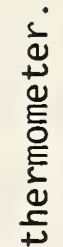

$\underline{a}$

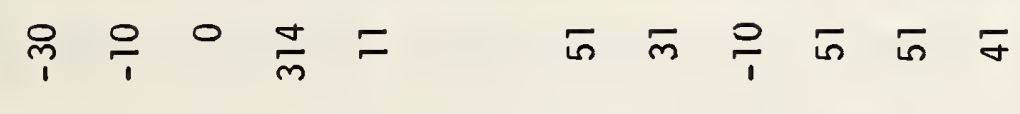

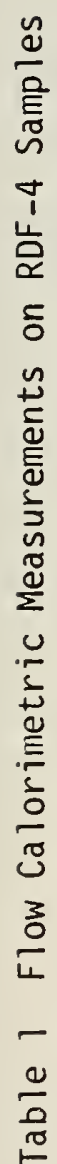

i

ก

$\underset{\frac{1}{ \pm}}{\frac{5}{2}}$

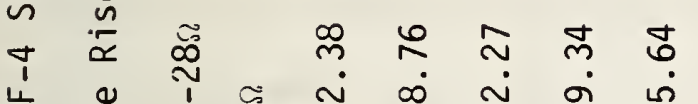

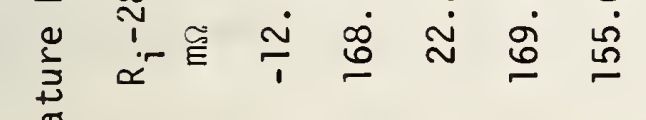

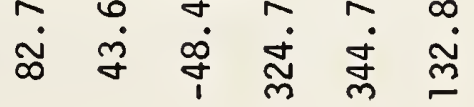

б

章

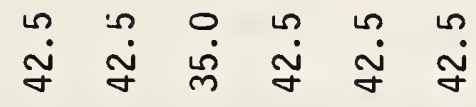

$\stackrel{\square}{ \pm}$

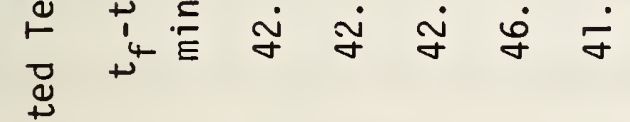

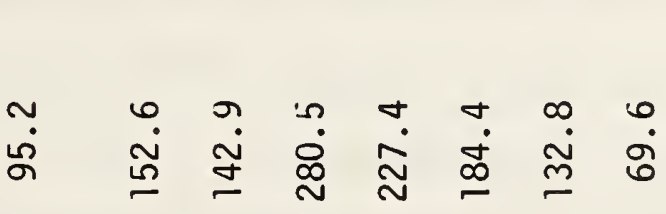

$\pm$

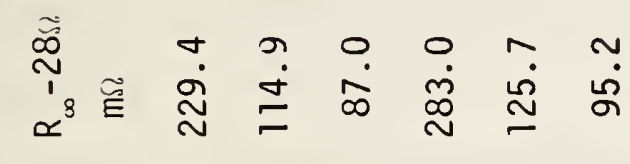

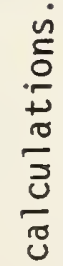

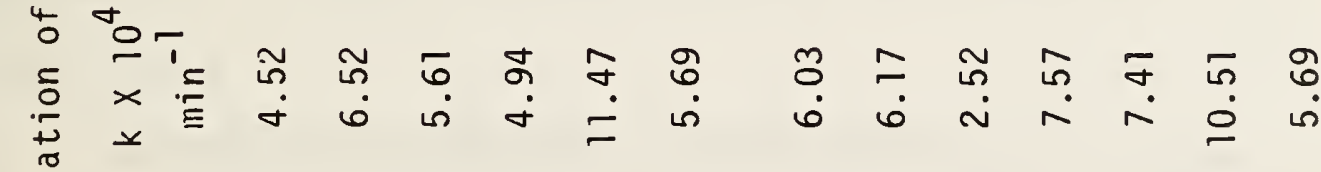
$\frac{3}{3}$

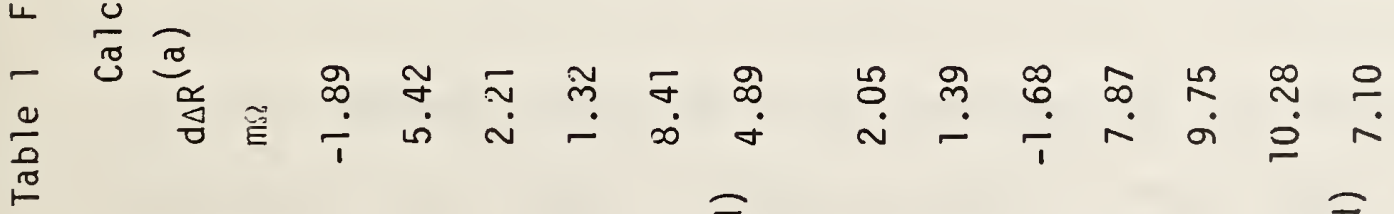

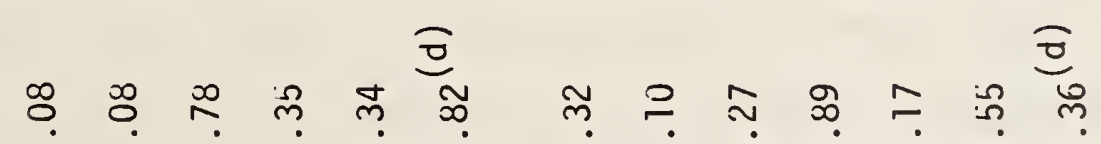

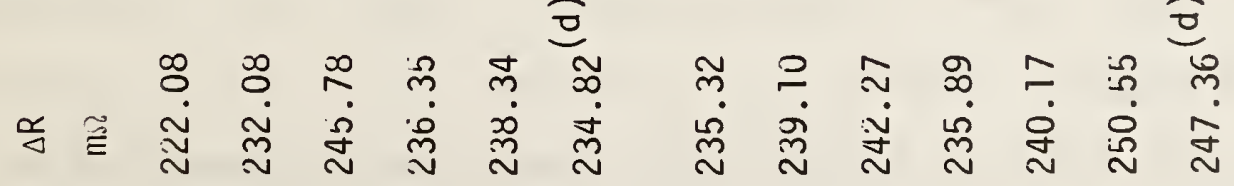


In eq. $4, k_{c}$ is the average cooling constant of the first nine experiments, and was calculated to be $5.69 \times 10^{-4} \mathrm{~min}^{-1}$. Adjusted values are listed in the second row of results for experiments 10 and 11 .

\subsection{Calculation of Enthalpy Changes}

The changes in state corresponding to each of the enthalpy changes in eq. 1 are given in succeeding sections." The calculations and the changes in state are based on the following assumptions:

a) The pressures of the gases entering and leaving the calorimeter are constant and differ negligibly from one atmosphere $(101.325 \mathrm{kPa})$; the pressure in the combustor at times $t_{i}$ and $t_{f}$ is one atmosphere. We have assumed that the gases (or gas mixtures) obey the perfect gas law.

b) Oxygen entering the calorimeter is pure except for the presence of water vapor and the moles of oxygen within the boundary of the calorimeter are the same at times $t_{j}$ and $t_{f}$.

c) In the combustion reaction, the nitrogen, sulfur, and chlorine of the RDF-4 sample that are not retained in the ash are completely converted to $\mathrm{N}_{2}(\mathrm{~g})$, $\mathrm{SO}_{2}(\mathrm{~g})$, and aqueous $\mathrm{HCl}$, respectively. The last species is assumed to be condensed completely into the water trap.

d) The only product due to incomplete combustion of the organic fraction of RDF-4 is CO.

e) The energy equivalent of the calorimeter is assumed to be the same at times $t_{j}$ and $t_{f}$.

f) The temperature, $T_{h}$, of the isothermal flow process (analogous to the isothermal bomb process of bomb calorimetry) is equal to $T_{j}$, the temperature of the calorimeter at time $t_{j}$.

g) The correction for viscous effects and kinetic energy losses of the flowing gases is negligible. 
3.3 Calculation of $\Delta \mathrm{H}_{\mathrm{st}}$ from Bomb Calorimetry

The change in state corresponding to $\Delta H_{s t}$ for the flow experiments is given by the equation for the standard flow reaction for RDF-4,

$$
\begin{aligned}
& {\left[\left(n_{1}+n_{2}\right) O_{2}+n_{3} H_{2} \mathrm{O}\right]\left(g, T_{i}\right)+1 \text { gram RDF-4 }\left(s, T_{i}\right)=} \\
& {\left[n_{5} \mathrm{CO}_{2}+n_{6} N_{2}+n_{8} S_{2}+n_{2} O_{2}\right]\left(g, T_{i}\right)+m^{\prime} \operatorname{gram} \text { Ash }\left(s, T_{i}\right)+} \\
& {\left[n_{9} \mathrm{HCl}+n_{10} \mathrm{H}_{2} \mathrm{O}\right]\left(\operatorname{soln}, T_{i}\right) ; P=1 \mathrm{~atm} .}
\end{aligned}
$$

In eq. $5,(g)$ refers to gas, (s) to solid, and (soln) to solution; $n_{1}$ is the number of moles of $\mathrm{O}_{2}$ required to convert one gram of RDF-4 having some residual moisture to $n_{5}$ moles of $\mathrm{CO}_{2}(\mathrm{~g}), \mathrm{n}_{6}$ moles of $\mathrm{N}_{2}(\mathrm{~g}), \mathrm{n}_{8}$ moles of $\mathrm{SO}_{2}(\mathrm{~g}), \mathrm{n}_{9}$ moles aqueous $\mathrm{HCl}$, and $n_{10}$ moles of liquid water. The quantities $n_{2}$ and $n_{3}$ are the moles of oxygen in excess of stoichiometry and water vapor that enter the calorimeter during the main period, respectively. $P=1 \mathrm{~atm}$ indicates the total pressure of products or reactants is one atmosphere.

The molar quantities $n_{1} n_{5}, n_{6}, n_{8}, n_{9}$ and $n_{10}$ are calculated from the elemental composition determined at the time of the bomb calorimetric measurements. The quantity $n_{10}$ is the most important. In the shortcut procedure of this work, ${ }^{n} 10$ minus the moles of water per gram RDF-4 remaining in the water trap is used to evaluate the largest of the correction term on the right side of eq. $1, \Delta H_{v}$. The values of $n_{10}$ were checked in the last two flow experiments, as discussed in section 3.6 .

The elemental composition determinations and the energy of combustion data deternined by bomb calorimetry are summarized in table 2. Results are listed for moisture-free RDF-4; composition is given in terms of mass fraction. Each lot was homogenized in a vee-blender, coned, and quartered. An elemental composition determination, two moisture determinations, and a single measurement of the energy of combustion were made using samples drawn from each quarter. The mass fraction of $H$ for Lot $A$ actually used in the computations is listed in parentheses and is discussed in section 3.6 . 
Table 2 Bomb Calorimetric Measurements on RDF-4 Samples

Elemental Composition (mass fraction, moisture free)

$\begin{array}{cccccccc}\text { Quarter } & \text { C } & \text { H } & \text { N } & \text { A } & \text { S } & \text { Cl } & \text { Ash } \\ & & & \text { Lot A } & & & & \\ 1 & .4273 & .0637 & .0079 & .2653 & .0047 & .0027 & .2283 \\ 2 & .4386 & .0645 & .0083 & .2601 & .0048 & .0029 & .2207 \\ 3 & .4259 & .0634 & .0077 & .2696 & .0048 & .0027 & .2259 \\ 4 & .4367 & .0629 & .0074 & .2596 & .0049 & .0030 & .2256 \\ \text { AVERAGE } & .4321 & .0636 & .0078 & .2637 & .0048 & .0028 & .2252 \\ & & . .0523)(\mathrm{a}) & & & & & \\ 1 & .4249 & .0546 & .0056 & .2546 & .0014 & .0035 & .2554 \\ 2 & .3925 & .0511 & .0049 & .3048 & .0013 & .0034 & .2420 \\ 3 & .3576 & .0462 & .0046 & .3529 & .0013 & .0034 & .2340 \\ 4 & .4174 & .0545 & .0044 & .2822 & .0013 & .0043 & .2353 \\ \text { AVERAGE } & .3981 & .0516 & .0049 & .2986 & .0013 & .0036 & .2418\end{array}$

(a) Value actually used, see section 3.6. 


\section{Table 2 (continued)}

\section{Energy of Combustion (moisture free)}

Quarter

Lot $A$

$\mathrm{HHV}_{2}(\mathrm{a})$

1

2

3

4

AVERAGE

Std. Dev. Mean

Imprecision $(\mathrm{b})$

$\mathrm{J} \cdot \mathrm{g}^{-1}$

17010

17087

16889

17054

17010

$\pm 43(.25 \%)$

$\pm 137(.80 \%)$
Lot $B$

HHV2

$\begin{array}{clc}\begin{array}{c}\text { Btu } \cdot 1 b^{-1} \\ 7313\end{array} & 17208 & \begin{array}{c}\text { Btu. } 1 \mathrm{~b}^{-1} \\ 7398\end{array} \\ 7346 & 17291 & 7434 \\ 7261 & 17268 & 7424 \\ 7331 & 17324 & 7448 \\ 7331 & 17273 & 7426 \\ \pm 18 & \pm 24(.14 \%) & \pm 10 \\ \pm 70 & \pm 76(.43 \%) & \pm 33\end{array}$

(a) HHV2: Energy of Combustion, moisture free; $1 \mathrm{~kJ} \cdot \mathrm{g}^{-1}=429.9226 \mathrm{Btu} \cdot 1 \mathrm{~b}^{-1}$.

(b) Calculated as 3.18 times the std. dev. of mean. 
To calculate the parameters in eq. 5 for the flow experiments, the following assumptions were made:

a) The four fractions of each lot are identical.

b) The ash in the bomb and flow reactions are identical (i.e. in mass fraction and chemical composition).

The results are summarized in table 3 for RDF-4 samples which have the appropriate residual moisture contents determined at the time of the flow measurements: $5.08 \pm 0.01$ and $3.96 \pm .01$ mass percent for Lot $A$ and Lot $B$, respectively.

To calculate $\Delta \mathrm{H}_{\text {st }}$ from HHV2 of the bomb measurements, the following additional assumptions were made:

a) The net correction to obtain HHV2 for the bomb reaction at atmospheric pressure from the measured HHV2 at 30 atmosphere pressure of oxygen is negligible.

b) The change in HHV2 or $\Delta H_{s t}$ for the flow reaction with temperature can be neglected.

The calculation of $\Delta \mathrm{H}_{\mathrm{st}}$ for both lots is summarized in table 4 . HHV2 is the average of the four fractions for each lot; the uncertainty is the imprecision corresponding to $95 \%$ confidence limit and three degrees of freedom. HHV2-HHV(AD) is the correction to convert one gram of dry RDF-4 to one gram of RDF-4 having the moisture contents appropriate to the flow experiments. The parameter $\Delta n R T$ is the pressure-volume correction to obtain $\Delta H_{s t}$ from HHV2. $\Delta \mathrm{n}$ is the sum of moles of $\mathrm{CO}_{2}(\mathrm{~g}), \mathrm{N}_{2}(\mathrm{~g})$, and $\mathrm{SO}_{2}(\mathrm{~g})$ minus the moles of stoichiometric $0_{2} \cdot \Delta \mathrm{n}$ is given in table 3 .

\subsection{Calculation of $\Delta \mathrm{H}_{\mathrm{ic}}$} $\Delta \mathrm{H}_{i c}$ was calculated for the change in state

$$
n_{4} \mathrm{CO}_{2}(g)=n_{4} \mathrm{CO}(g)+\left(n_{4} / 2\right) O_{2}(g) ; T_{i} ; P=1 \mathrm{~atm}
$$


Table 3. Standard Flow Reaction

Composition of one gram of RDF-4

formula: $\left[\begin{array}{lllllll}\mathrm{C}_{a} & \mathrm{H}_{b} & \mathrm{~N}_{c} & \mathrm{O}_{d} & \mathrm{~S}_{e} & \mathrm{Cl}_{f}\end{array}\right]\left(\mathrm{H}_{2} \mathrm{O}\right)_{h}$ (m' grams Ash)

Symbol mmol, Lot A

a $\quad 34.15$

b

c

d

e

$\mathrm{f}$

h

$m^{\prime}$ $59.9(49.6)^{(a)}$

0.53

15.64

0.15

0.075

2.82

0.2138 mmol, Lot B

31.83

4.92

0.34

17.92

0.039

0.10

2.20

0.2322

Values of Quantities in the Standard Flow Reaction, eq. 5.

Symbo

$\mathrm{n}_{1}$

$n_{5}$

$n_{6}$

$n_{8}$

$\mathrm{n}_{9}$

${ }^{n} 10$

$\Delta n^{(c)}$ (see text)

m"
Species

$0_{2}(g)$

$\mathrm{CO}_{2}(\mathrm{~g})$

$\mathrm{N}_{2}(\mathrm{~g})$

$\mathrm{SO}_{2}(\mathrm{~g})$

$\mathrm{HCL}$ (aq)

$\mathrm{H}_{2} \mathrm{O}$ (aq)

Ash mmo1, Lot $A$

$41.44(38.88)^{(a)}$

34.15

0.27

0.15

0.075

$32.73(26.6)^{(a)}$

$-6.87(-4.29)^{(a)}$

0.2138 mmol, Lot B

35.18

31.83

0.17

0.039

0.10

26.75

$-3.14$

0.2322

(a) Value actually used, see section 3.6.

(b) $n_{1}=a+e+b / 4-f / 4-d / 2, n_{5}=a, n_{6}=c / 2, n_{8}=e, n_{g}=f, n_{10}=b / 2+h-f / 2$.

(c) $\Delta n=n_{5}+n_{6}+n_{8}-n_{1}$ 


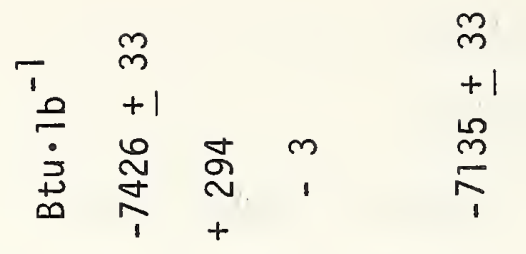

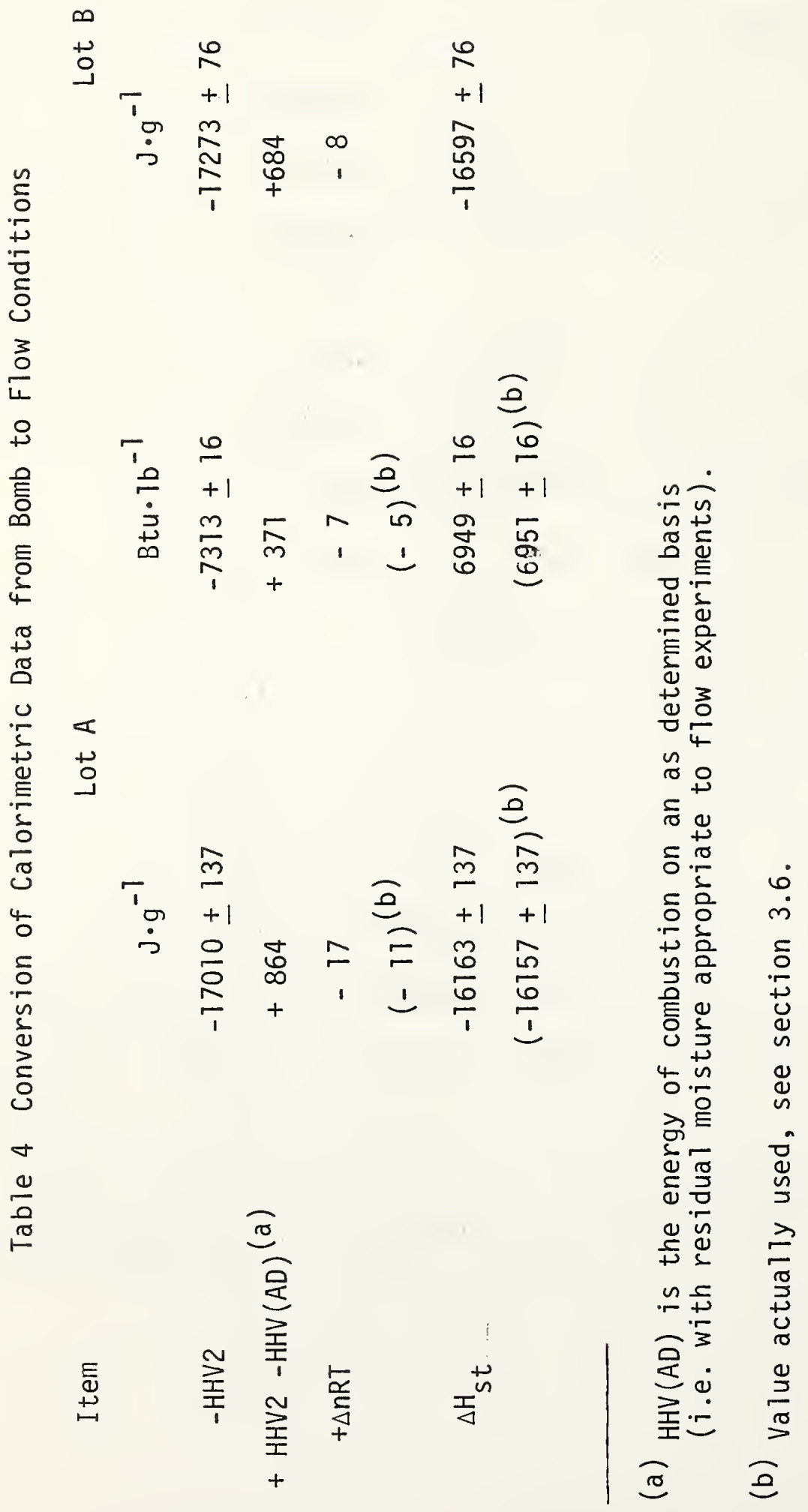


where $n_{4}$ is the number of moles of $C 0$ per gram RDF-4. The enthalpy change per mole of $\mathrm{CO}$ was taken as $298 \mathrm{~kJ} \cdot \mathrm{mol}^{-1}$ from reference 4 . The value of $\mathrm{n}_{4}$ was calculated using the measured volume flow rate of the gases entering the co detector and the pressure and temperature of the gases leaving the detector. During a combustion run, the latter parameters were constant to $2 \%$ for the flow rate, 0.2 to $0.5 \mathrm{kPa}$ for the pressure, and $0.2{ }^{\circ} \mathrm{C}$ for the temperature. The CO detector was calibrated using known concentrations of $0.1,0.3,0.6,1$, and $2 \mathrm{~mol} \% \mathrm{CO}$ in nitrogen.

\subsection{Calculation of $\Delta \mathrm{H}_{\mathrm{g}}$}

The reaction corresponding to $\Delta H_{g}$ is

$$
[X]\left(g, T_{i n}\right)+[Y]\left(g, T_{i}\right)=[X]\left(g, T_{i}\right)+[Y]\left(g, T_{e x}\right)
$$

where

$$
x=\left(n_{1}+n_{2}-n_{4} / 2\right) O_{2}+n_{3} H_{2} O
$$

and

$$
Y=\left(n_{5}-n_{4}\right) \mathrm{CO}_{2}+n_{4} \mathrm{CO}+n_{6} \mathrm{~N}_{2}+n_{8} \mathrm{SO}_{2}+n_{2} \mathrm{O}_{2}+\left(n_{3}+n_{7}\right) \mathrm{H}_{2} \mathrm{O}
$$

In eq. $7, T_{i n}$ and $T_{e x}$ are the temperatures of the gases entering (in) and leaving (ex) the calorimeter, respectively. $T_{i}$ corresponds to $T_{h} \cdot n_{7}$ is the moles of water vapor leaving the calorimeter in excess of the amount, $n_{3}$, entering during the main period. $\Delta H_{g}$ was calculated using eq. 8.

$$
\begin{aligned}
& \Delta H_{g}=\Delta H_{g 1}+\Delta H_{g 2}+\Delta H_{g 3} ; \\
& \Delta H_{g l} \cong\left(n_{2} C_{2}+n_{3} C_{3}\right)\left(T_{e x}-T_{i n}\right) ; \\
& \Delta H_{g 2} \cong n_{1} C_{2}\left(T_{i}-T_{i n}\right) ; \\
& \Delta H_{g 3} \cong\left(n_{7} C_{3}+n_{5} C_{5}\right)\left(T_{e x}-T_{i}\right) .
\end{aligned}
$$

$C_{2}, C_{3}$, and $C_{5}$ are the molar heat capacities of gaseous oxygen, water, and $\mathrm{CO}_{2}$ and were taken as the values given in reference 4 at $25^{\circ} \mathrm{C}$. The approximate equalities assume $n_{4}$ and $n_{6}$ are negligible. $T_{\text {in }}$ and $T_{\text {ex }}$ were assumed to equal 
respectively, the room temperature, $T_{r}$, and $T$, the temperature corresponding to the average resistance, $\bar{R}$, of the platinum thermometer over the main period listed in the last column of table 1. The numerical value of $\Delta H_{g l}$ is about $93 \%$ of $\Delta \mathrm{H}_{e}$ in our experiments. Thus $\Delta \mathrm{H}_{\mathrm{g}}$ is quite insensitive to the value selected for $T_{h}$ (i.e. here $T_{j}$ ).

The values for $n_{1}$ and $n_{5}$ were taken from table 3 . The value of $n_{2}$ was calculated from $n_{1}$ and $\left(n_{1}+n_{2}\right)$. The value of $\left(n_{1}+n_{2}\right)$ was calculated from the sum of the measured volume flow rates of the primary and secondary oxygen. $n_{3}$ was calculated assuming the inlet oxygen in experiments 1 through 9 was saturated with water vapor at room temperature. Values of $n_{7}$ were calculated as discussed in section 3.6 and are given in table 6 . Temperature differences were calculated using the last three columns of table 1 and the conversion factor of $9.903 \Omega$ (ohms) difference equals $1 \mathrm{~K}$ difference in temperature.

\subsection{Calculation of $\Delta H_{V}$}

The change in state corresponding to $\Delta H_{v}$ is

$$
\begin{aligned}
& Z\left(\operatorname{soln}, T_{j}\right)+\left(n_{7}+n_{3}^{i}-n_{3}^{f}\right) H_{2} O\left(g, T_{i}\right)= \\
& {\left[Z+\left(n_{7}+n_{3}^{i}-n_{3}^{f}\right) H_{2} O\right]\left(\operatorname{soln}, T_{j}\right) ; P=1 \mathrm{~atm}}
\end{aligned}
$$

where $Z$ is defined as

$$
Z=\mathrm{n}_{8} \mathrm{H}_{2} \mathrm{SO}_{4}+\mathrm{n}_{9} \mathrm{HCl}+\mathrm{n}_{10} \mathrm{H}_{2} \mathrm{O} \text {. }
$$

In eq. $9, n_{7}$ is the net moles of water vaporized from the calorimeter during the main period. $n_{3}{ }^{j}$ and $n_{3}{ }^{f}$ are moles of water vapor present in the calorimeter at times $t_{i}$ and $t_{f}$, respectively. $n_{3}{ }^{i}-n_{3}^{f}$ was assumed to be negligible in comparison to $n_{7}$. 
$\Delta H_{v}$ was calculated using

$$
\Delta \mathrm{H}_{\mathrm{v}}=\mathrm{n}_{7} 44.011 \mathrm{~kJ} \cdot \mathrm{mol}^{-1}
$$

which assumes the solutions in eq. 9a are dilute.

The value for $n_{7}$ was first calculated in the shortcut procedure from the values $n_{10}$, the moles of water produced per gram RDF-4, given in table 3 and $n_{10^{-n}}$ calculated by eq. 11:

$$
n_{10}-n_{7}=\Delta w(18.0154 \cdot m)
$$

$\Delta \mathrm{W}$ is the increase of the mass of the water trap and $m$ is the mass of the RDF-4 sample. The quantity of water vaporized during the final drift period in experiments 1 through 9 was neglected since the inlet oxygen was saturated. In experiments 10 , and 11 , a correction, $\Delta n_{7}$, was subtracted from $n_{10}-n_{7}$ for this effect since the inlet oxygen was dry. $\Delta n_{7}$ was calculated from:

$$
\Delta n_{7}=\left(k-k_{c}\right)\left(R_{f}-R_{j}\right) E \Delta t_{f} /(44017 \cdot m)
$$

In eq. $12, k$ is the observed cooling constant and $k_{c}$ is the average cooling constant for runs 1 through $9 . R_{f}$ and $R_{j}$ are the resistances of the platinum thermometer at the end $(f)$ and beginning ( $i$ ) of the main period. $E$ is the energy equivalent of the calorimeter in units of $\mathrm{J} \cdot \Omega^{-1} \cdot \Delta t_{f}$ is the length of the final drift period in minutes. Again, $m$ is the mass of the RDF-4 sample. The values of $n_{10^{-n_{7}}}$ are listed in column two of table 5 .

The values of $n_{10}$ for Lot $A$ and Lot $B$ were checked in experiments $10 A$ and $11 \mathrm{~B}$, respectively, by passing the product gases through a drying trap consisting of tubes containing anhydrous $\mathrm{CaSO}_{4}$ (Drierite) instead of the water bubbler. Since the inlet oxygen was dry in these flow experiments, $n_{7}$ can be determined directly from the increase in mass of the drying trap and $n_{10}$ was calculated from $n_{7}$ plus the increase in the mass of the water condenser in the calorimeter. The results were 
$n_{10}$ (flow expts.), mmol
$26.6 \pm 0.4$
$26.9 \pm 0.4$
$32.7 \pm 0.5$
$26.8 \pm 0.5$

$n_{10}($ table 3$)$, mmol

The uncertainty in $n_{10}$ determined in the flow experiments is an estimate. The uncertainty in $n_{10}$ from table 3 is based on the imprecision at the $95 \%$ confidence level of the four measurements of the hydrogen content for each log given in table 2. The two values for Lot $B$ agree within their combined uncertainties but those for Lot $A$ do not. The value of $n_{10}$ measured for Lot $A$ in the flow experiments was taken as the correct value for reasons given below.

First, the efficiency of the water trap, calculated as $\left(n_{10}-n_{7}\right) / n_{10}$, should be independent of whether the sample that is being burned is from Lot A or Lot B. Comparison of the entries in the third column of table 5 (e.g. compare $1 A$ with $2 B$, $4 A$ with the mean of $3 B$ and $5 B$, the mean of $6 A$ and $7 A$ with the mean of $8 B$ and $9 B$, and $10 A$ with $11 B$ ) shows the trap efficiency is consistently lower for Lot $A$ as compared to Lot $B$. Hence, $n_{10}$ for Lot $A$ is too large. An equivalent and clearer result of demonstrating the same result is to assume that, in experiments 1 through 9 , the pressure of water vapor, $\mathrm{P}\left(\mathrm{H}_{2} \mathrm{O}\right.$, out), in the gases leaving the calorimeter is equal to the vapor pressure at the temperature $T_{\text {ex }}$ (see section 3.5 ). Then, the pressure, $P\left(\mathrm{H}_{2} \mathrm{O}\right.$, in), of water vapor in the gases entering the calorimeter can be calculated from $n_{7}{ }^{*} \cdot P\left(H_{2} O\right.$, in ) should be near the vapor pressure, $P_{\text {sat }}$,

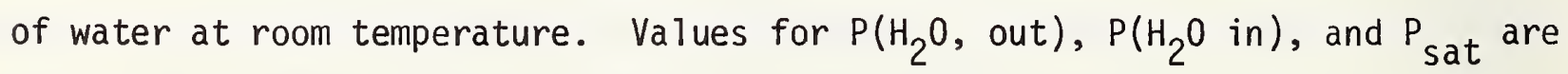
listed in columns 4 through 6 of table 5. Comparison of columns 5 and 6 shows that $P\left(\mathrm{H}_{2} \mathrm{O}\right.$, in $) / P_{\text {sat }}$ is near one for Lot $B$ but is about $2 / 3$ for Lot $A$. If $n_{10}$ for Lot $A$ is changed from 32.6 to $27.6 \mathrm{~mol}$, the trap efficiencies and $P\left(\mathrm{H}_{2} \mathrm{O}\right.$, in $) / P_{\text {sat }}$ of Lot $A$ and Lot $B$ are about the same. This can be seen from the revised trap efficiencies and pressure ratios given in the parentheses next to columns 3 and 4 of table 5 , respectively.

\footnotetext{
* $\mathrm{P}\left(\mathrm{H}_{2}\right)$, in $)$ was calculated from

$$
P\left(H_{2} 0, \text { in }\right) \frac{V}{R T}+n_{7}=\left(P H_{2} 0, \text { out }\right) \frac{V}{R T} \text { ex } ; V=V\left(t_{f}-t_{i}\right) ;
$$

$V$ is the measured total volume flow rate.
} 


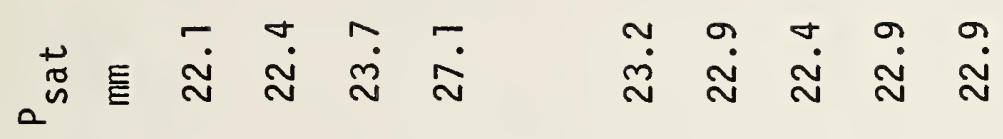

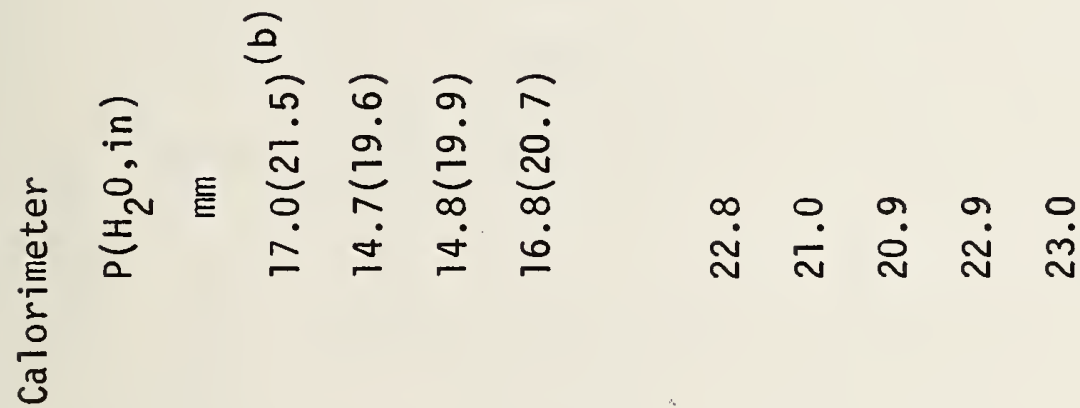

$\stackrel{\square}{ \pm}$

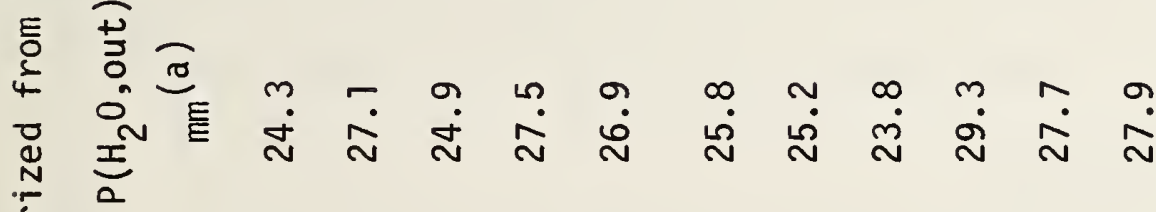

寒

离 要

प.

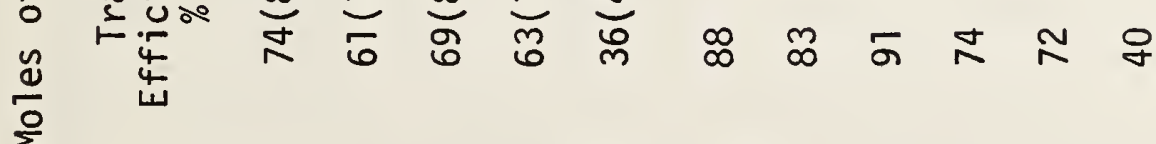

这

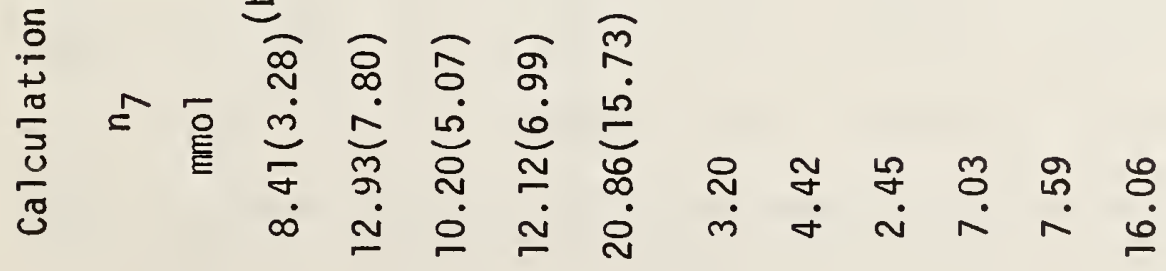

เ

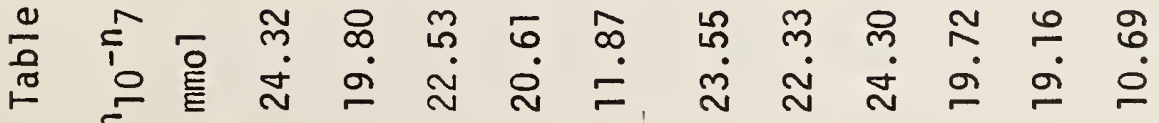

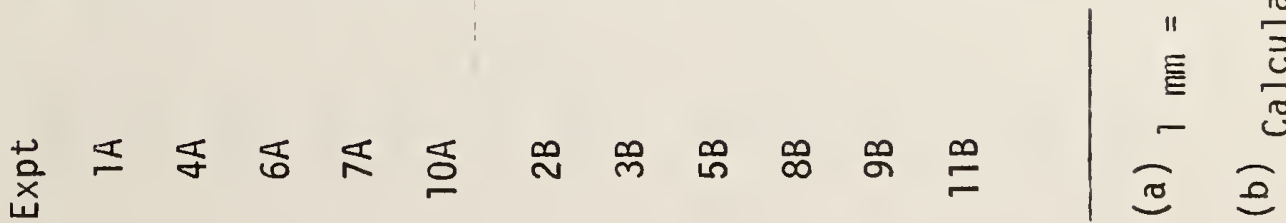


The second reason for adopting $27.6 \mathrm{mmol}$ for $n_{10}$ of Lot $A$ was that a redetermination of the hydrogen content of the remaining portion of Lot $A$ (part of quarter 2, see table 2) gave a value for $n_{10}$ of $25.0 \pm 3.5 \mathrm{mmol}$. This agrees with the result of experiment 10A but disagrees with the values listed in table 3. The carbon content of the same part of Lot A was $39.79 \pm 5.39$ mass \% (dry basis) which agrees with that given in table 3, $43.21 \pm 1.03$ mass \% Thus, segregation cannot account for the discrepancy in the hydrogen concentration.

Revised values for the mass percent of hydrogen, $n_{1}, \Delta n$, and $\Delta H_{s t}$ for Lot $A$ based on $n_{10}=27.6 \mathrm{mmol}$ are given in parentheses of tables 2 through 4.

\subsection{Calculation of $\Delta \mathrm{H}_{i g n}$}

$\Delta H_{i g n}$ was assumed to correspond to the change in state

$(7 / 2) \mathrm{O}_{2}(\mathrm{~g})+\mathrm{Fe}(\mathrm{c})=(1 / 2)\left[\mathrm{Fe}_{2} \mathrm{O}_{3}(\mathrm{~s})+\mathrm{Fe}_{3} \mathrm{O}_{4}(\mathrm{~s})\right]$

Eq. 13 assumes no interaction with the ash and that fuse wire is completely oxidized. $\Delta H$ for eq. 13 was taken as $6.66 \mathrm{~kJ} \cdot \mathrm{g}^{-1}$ from reference 4 .

\subsection{Summary of Calculations}

Table 6 lists the values of moles per gram of RDF-4 of $\mathrm{CO}(\mathrm{g}), \mathrm{n}_{4}$, excess oxygen entering the calorimeter, $n_{2}$, moles of water vapor entering the calorimeter, $n_{3}$, and net moles of water vaporized, $n_{7}$.

Table 7 summarizes the calculations of $E$ and $-\Delta H_{s t}$. The estimated value of $\Delta H_{i c}$ for experiment $5 B$ is the average $\Delta H_{i c}$ of the other experiments with Lot $B$. 4.0 Systematic Error Analys is of 25 Gram Flow Experiments.

The larger systematic errors in the calculated results are identified and their magnitudes are estimated in the following sections. Most of the estimates are based on auxiliary experimental measurements which are also described. 


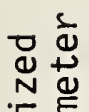

$\therefore$.

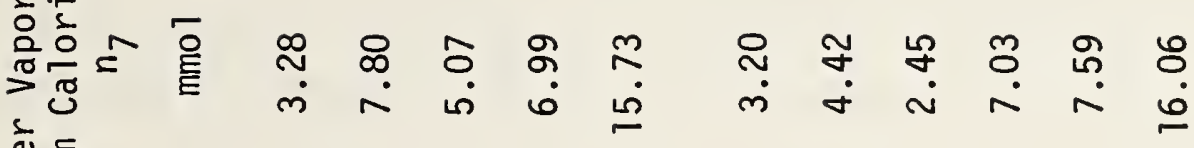

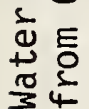

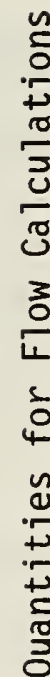

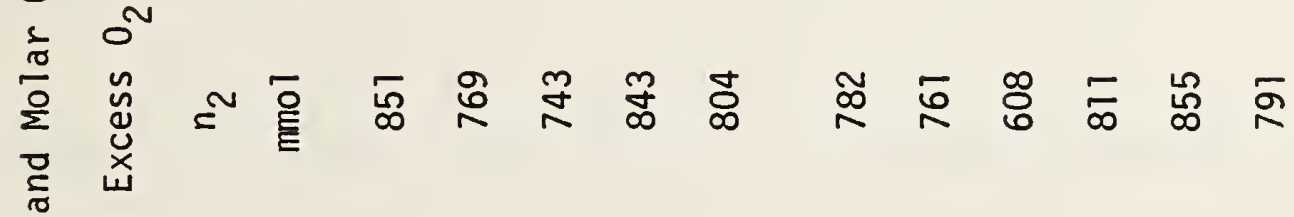

n

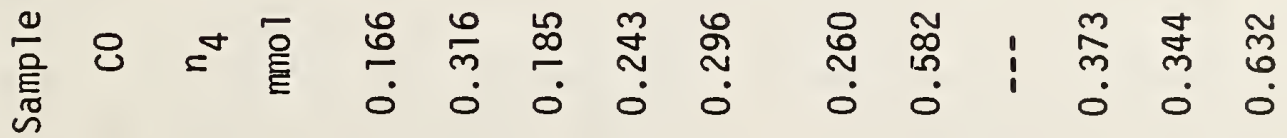

$\omega$

$\frac{\omega}{\circ} \frac{0}{\circ} \tilde{\circ}$

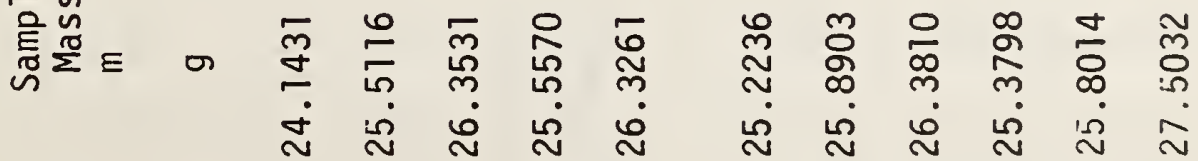

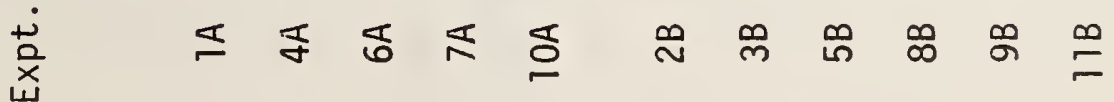



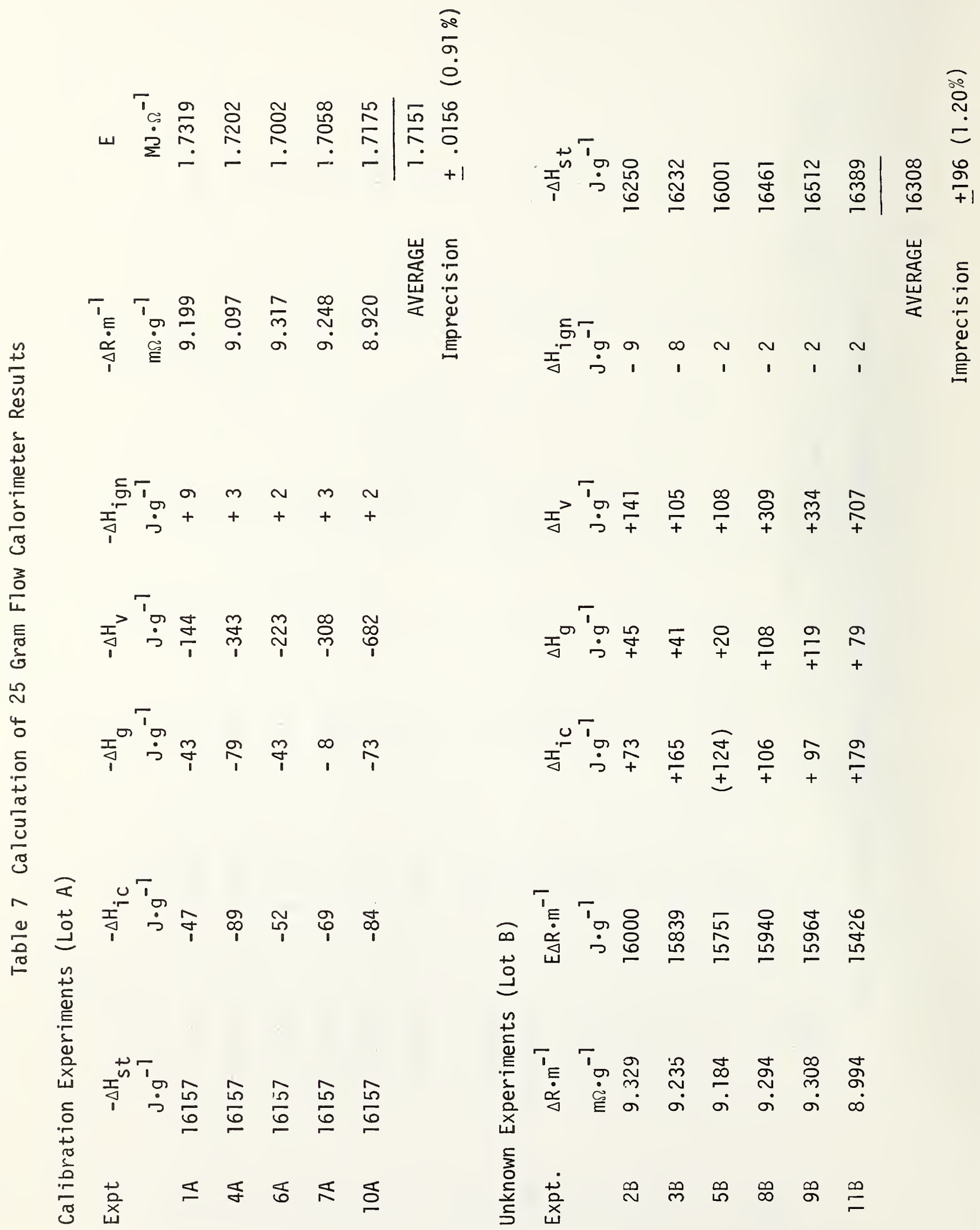


\subsection{Errors in $\Delta R$}

The errors in $k$ and $R_{\infty}$ caused by allowing gases to enter and leave the calorimeter during drift periods are negligible so long as no water is vaporized from the calorimeter. (The estimated error in $k$ is $0.03 \times 10^{-4} \mathrm{~min}^{-1}$.) The calculations assume that no water is vaporized from the calorimeter during the final drift period when the inlet oxygen was saturated. To estimate the error caused by deviations from this assumption, $k$ of eq. 3 was determined when no gases were allowed to enter or leave the calorimeter. The validity of eq. 3 was also checked. This was done as follows.

An electrical heater was installed in the provisional calorimeter and a series of drift rates, $d R / d t$, were determined for eight values of $R$ between 27.99 and $29.56 \Omega$ without opening the calorimeter. Measurements were made over a period of two days at a constant room temperature of $24.4{ }^{\circ} \mathrm{C}\left(R_{r}\right.$ defined in table 1 was $28.031 \Omega)$. Provided drift rates were determined thirty or more minutes after the heater was turned off, all the values of $d R / d t$ lay on a straight line with a standard deviation of $0.4 \times 10^{-5} \Omega / \mathrm{min}$. A least square fit of the data gave

$$
(d R / d t) \times 10^{5}=6.22 \pm 0.72-(36.1 \pm 2.8)(R-28)
$$

Uncertainties are imprecisions at the $95 \%$ confidence level for eight measurements.

The results indicate that eq. 3 is valid in the preliminary flow experiments. The results also show that effect of vaporization of water during the final drift period on $k$ and $R_{\infty}$ in experiments 1 to 9 cannot be neglected since the average $k$ for these experiments is $5.69 \times 10^{-4} \mathrm{~min}^{-1}$. Correction of all experiments using eq. 4 with $k_{c}$ equal to $3.6 \times 10^{-4}$ min $^{-1}$ increases $E$ by $0.5 \%$ and decreases $-\Delta H_{s t}$ for lot $B$ by $0.6 \%$. To be consistent, an additional correction must be done on $\Delta H_{v}$ since vaporization of water cannot be neglected during the final drift period when the inlet oxygen is saturated. The correction is estimated in section 4.5 . The combined effect is to increase $E$ by $0.7 \%$ and decrease $-\Delta H_{\text {st }}$ for Lot $B$ by $0.7 \%$. 


\subsection{Errors in $\Delta H_{s t}$}

The measured millequivalents of acid per gram (meq $\cdot \mathrm{g}^{-1}$ ) of RDF-4 sample contained in the water trap are listed in the first column of table 8 . From table 3 , one would expect 0.08 for Lot $A$ and 0.10 for Lot B. Estimated correction of the experimental results for vaporization of acid (by multiplying each by $n_{10} /\left(n_{10}-n_{7}\right)$ from table 5) yields the results given in the second column of table 8. The measured value of the acid content of the water in the bubbler through which the cumulative product gases of experiments 6 through 9 were passed was $0.064 \mathrm{meq} \cdot \mathrm{g}^{-1}$. This is in reasonable agreement with the estimated value of $0.073 \mathrm{meq} \cdot \mathrm{g}^{-1}$ which was computed as the sum of the values in the third column in table 8 minus the sum of those in the second column for these experiments. However, since the values given in the second column of table 8 for experiments 10 and 11 grossly exceed the predicted results, the agreement must be regarded as fortuitous. We concluded that 1) most of the oxidized sulfur is $\left.\mathrm{SO}_{2}(g), 2\right)$ most of the aqueous acid is probably $\mathrm{HCl}$, and 3) the correction for the small but unknown amount of aqueous $\mathrm{H}_{2} \mathrm{SO}_{4}$ is negligible.

The possibility that the chemical composition of the ash produced in the bomb reaction is different from that of the flow reaction is not reflected in differences in the mass fraction of the ash contents. The mass fraction of the ash determined by weighing of the residue in the bomb experiments, flow experiments, and ASTM test methods corrected to the moisture contents of the flow work are given in Table 8 . 
Table 8 Acid Content of Water Trap

$\begin{array}{rcc}\text { Expt. } & \begin{array}{c}\text { Acid } \\ \mathrm{meq} \cdot \mathrm{g}^{-1}\end{array} & \begin{array}{c}\text { Acid/Trap Efficiency } \\ \text { (b) } \\ \text { IA }\end{array} \\ \text { meq } \cdot \mathrm{g}^{-1}\end{array}$

(a) meq = milliequivalents

(b) See table 5 
ASTM Test ${ }^{(a)}$

$$
0.2138 \pm 0.0047
$$

$0.2322 \pm 0.0148$

Combustion Bomb

$0.1992 \pm 0.0048$

$0.2363 \pm 0.0035$

Flow Calorimeter

$0.2098 \pm 0.0074$

$0.2301+0.0028$

Uncertainties are imprecisions at the $95 \%$ confidence level. The results agree within their imprecisions.

(a) ASTM Test D3173-73

A check was made for possible changes or inhomogeneity in the RDF-4 samples between the time when the original composition determinations and bomb calorimetric experiments were made and flow calorimetric experiments were carried out. After the flow experiments, ash and moisture contents of each lot were determined before and after mixing each lot in a vee-blender. The mass fraction results of the ASTM test were as follows:

Lot $A$

Before mixing

After mixing
Ash

0.2135

0.0469

0.2133

0.0464
Lot B

0.2317

0.0369

0.2215

0.0354

Ash contents have been corrected to the moisture contents of the flow work for comparison with previous results. The only significant difference is in the Lot $B$ ash content which indicates that some segregation of Lot B may have occurred. This possibility is also reflected in the large uncertainty associated with the original ash determinations on Lot B (see above). Differences between these moisture contents and those used in the flow work (i.e. 0.0508 for Lot $A$ and 0.0396 for Lot B) suggested the assignment of an estimated systematic error of 0.005 to the mass fraction of moisture for each lot in the flow work. 


\subsection{Errors in $\Delta \mathrm{H}_{\mathrm{ic}}$}

Maximum errors of $5 \%$ in the gas flow rate and $4 \%$ in the fractional content of CO in the product gases produce errors of $0.03 \%$ and $0.02 \%$, respectively, in $E$. The net effect on $-\Delta H_{s t}$ for Lot $B$, if the error is the same in all experiments, is negligible.

The average loss in mass of the ash from the flow experiments due to heating with the flame of an oxygen-gas torch was $0.071 \%$ of the initial sample mass. The species which vaporized or were oxidized were not identified. After several experiments, black smudges resembling "soot" were observed on the quartz plate and/or crucible; these disappeared upon flaming.

The residual carbon content of the heated ash was determined to be $0.008 \%$ of the initial sample mass.

Traces of brownish material resembling "tars" were found on the exit port of the thermal shield after each combustion run, the mass was less than $0.001 \%$ of the initial sample mass.

The total solid products of incomplete combustion constitute $0.08 \%$ of the initial sample mass. Treated as unburned combustibles in RDF-4, E would increase by $0.10 \%$. If the solids are treated as pure carbon, $E$ increases by $0.14 \%$. In either case the net effect on $-\Delta H_{s t}$ for Lot $B$ is negligible.

\subsection{Errors in $\Delta H_{\mathrm{g}}$}

A maximum error of $5 \%$ in the inlet gas flow rate introduces an error of $0.02 \%$ in $E$. Combined with the flow rate effect on $\Delta H_{i c}$, a $5 \%$ error in flow rates introduces an error of $0.05 \%$ in $E$. The net effect on $-\Delta H_{s t}$ for the same error in $a 11$ experiments is negligible.

Measurement of $T_{\text {ex }}-T_{r}$ and $T_{i n}-T_{r}$, where $T_{r}$ is the room temperature were made in experiments 5,6, 9, and 11 using Type $k$ thermocouples. Analys is of the data showed that during drift periods eqs. 14 apply. 


$$
\begin{aligned}
& T_{\text {in }}-T_{r} \simeq-0.17+0.26\left(T-T_{r}-0.17\right) \\
& T_{\text {ex }}-T \simeq-0.13\left(T-T_{r}-0.17\right)
\end{aligned}
$$

$T$ is the observed temperature of the calorimeter water. Deviations from eq. 14b during the main period were less than $0.1 \mathrm{~K}$ except for temporary changes in thermocouple voltage that seem to be associated with evaporation and/or condensation of moisture on the thermocouple junction. The uncertainty in the expressions on the right side of eqs. 14 is unknown but appreciable. In any event, recalculation of $\Delta H_{g l}$ for all experiments using eqs. 14 increases $E$ by $0.18 \%$ and decreases $-\Delta H_{s t}$ for Lot $B$ by $0.26 \%$.

\subsection{Errors in $\Delta \mathrm{H}_{\mathrm{V}}$}

The correction for the error made in assuming that the water vaporized during the final drift period is negligible when the inlet oxygen is saturated was calculated using eq. 10 and the drift data given in section 4.1. E increases by $0.17 \%$ and $-\Delta H_{\text {st }}$ for Lot $B$ decreases by $0.13 \%$.

\subsection{Summary of Error Analysis}

A summary of the effect of the errors expressed in terms of their percentage effect on the energy equivalent, $E$, and the energy of combustion, $-\Delta H_{s t}$, of Lot $B$ is given in table 9. The parentheses after the error source enclose the section number where the analysis is given. Below each error source, variation contributions are itemized. The percent effect of each error contribution to $-\Delta H_{S t}$, given in column three, was computed additively.

The total systematic error in $-\Delta \mathrm{H}_{\mathrm{st}}$ is $-1.0 \pm 1.0 \%$. The systematic error associated with the provisional calorimetric assembly is considered to be $-2.6 \%$ (item $4 . b$ ). 
Table 9. Systematic Error Analysis of 25 Gram Flow Experiments

EFFECT

Source of Error

$\mathrm{E}$

$(\%)$

$-\Delta H_{s t}$

$(\%)$

1. $\Delta R(4.1)$

no vaporization of water

$+0.5$

$-0.6$

in final drift (inlet $\mathrm{O}_{2}$ satd)

2. Sample mass

moisture content

$\pm 0.5$

$\pm 1.0$

3. $\Delta \mathrm{H}_{\mathrm{ic}}(4.3)$
a) Co concentration(a)
$<+0.02$
0.0
b) flow rate $(\mathrm{b})$.
$< \pm 0.03$
0.0
c) complete combustion
$+0.1$
0.0
to $\mathrm{CO}$ and $\mathrm{CO}_{2}$

4. $\Delta H_{g}(4.4)$
a) flow rate ${ }^{(b)}$
b) $T_{\text {in }}=T_{r}, T_{e x}=T$

$\leq \pm 0.02$

0.0

$+0.18$

5. $\Delta H_{v}(4.5)$

no vaporization of water in final drift (inlet $\mathrm{O}_{2}$ satd)

(a) Assumes error in co concentration of $\pm 4 \%$.

(b) Assumes error in product gas flow rate of $\pm 5 \%$. 


\section{Results of 25 Gram Flow Experiments and Conclusions}

A summary of the results is listed in table 10. In the calibration experiments, $\Delta H_{s t}$ is the enthalpy change for the standard flow reaction for one gram of RDF-4 calculated from the results of combustion bomb measurements. Results are expressed in terms of the mass of sample with moisture contents of the actual calibration experiments. $\Delta \mathrm{H}_{\mathrm{ic}}$ is the average correction per gram of RDF-4 for incomplete combustion of all CO to $\mathrm{CO}_{2} \cdot \Delta \mathrm{H}_{\mathrm{g}}$ is the average correction per gram of RDF-4 for the net heat transport by oxygen entering and gaseous products leaving the calorimeter at some temperature different than the temperature of the standard flow reaction (we assumed this temperature to be the initial calorimeter temperature). $\Delta H_{v}$ is the average correction for the heat of vaporization of water formed in the combustion that is lost from the calorimeter. $\Delta H_{i g n}$ is the average of the ratio of the enthalpy of the combustion of the iron fuse wire used to ignite the RDF-4 sample divided by the mass of the RDF-4 sample. $\Delta R / m$ is the average ratio of temperature rise, expressed in ohms (i.e. $0.1 \Omega \sim 1^{\circ} \mathrm{C}$ ), of the calorimeter corrected for heat leak divided by the mass of the sample, $m$.

$E$ is the mean energy equivalent of the calorimeter and is calculated from the basic measurement equation:

$$
\frac{E \Delta R}{m}=-\left(\Delta^{H} s t+\Delta H_{i c}+\Delta H_{g}+\Delta H_{v}+\Delta H_{i g n}\right)
$$

The imprecision is the product of the standard deviation of the mean and the "Student $t$ " factor for four degrees of freedom at the $95 \%$ confidence level. 
Table 10 Summary of 25 Gram Flow Results

Calibration Experiments (Lot A)

$\begin{array}{lcc}+\Delta H_{S t} & -16157 \mathrm{~J} \cdot \mathrm{g}^{-1} & \left(-6946 \mathrm{BTU} \cdot 1 \mathrm{~b}^{-1}\right) \\ +\Delta H_{i C} & +68 \mathrm{~J} \cdot \mathrm{g}^{-1} & \left(+29 \mathrm{BTU} \cdot 1 \mathrm{~b}^{-1}\right) \\ +\Delta H_{g} & +49 \mathrm{~J} \cdot \mathrm{g}^{-1} & \left(+21 \mathrm{BTU} \cdot 1 \mathrm{~b}^{-1}\right) \\ +\Delta \mathrm{H}_{\mathrm{V}} & +340 \mathrm{~J} \cdot \mathrm{g}^{-1} & \left(+146 \mathrm{BTU} \cdot 1 \mathrm{~b}^{-1}\right) \\ +\Delta H_{i g n} & -4 \mathrm{~J} \cdot \mathrm{g}^{-1} & \left(-2 \mathrm{BTU} \cdot 1 \mathrm{~b}^{-1}\right) \\ \Delta \mathrm{R} \cdot \mathrm{m}^{-1} & 9.156 \mathrm{~m} \Omega \cdot \mathrm{g}^{-1} & \left(4.153 \Omega \cdot 1 \mathrm{~b}^{-1}\right) \\ \mathrm{E} & 1.7151 \mathrm{MJ} \cdot \Omega^{-1} & \left(1608 \mathrm{BTU} \cdot \Omega^{-1}\right)\end{array}$

imprecision

$$
\pm .0156(0.91 \%) \pm 15
$$

Unknown Experiments (Lot $B$ )

$\Delta R \cdot m^{-1}$

$9.224 \mathrm{~m} \Omega \cdot \mathrm{g}^{-1}$

$\left(4.184 \Omega \cdot 1 b^{-1}\right)$

$E \Delta R \cdot m^{-1}$

$+15,820 \mathrm{~J} \cdot \mathrm{g}^{-1}$

$\left(+6801 \mathrm{BTU} \cdot 1 \mathrm{~b}^{-1}\right)$

$\Delta \mathrm{H}_{\mathrm{ic}}$

$+\quad 124 \mathrm{~J} \cdot \mathrm{g}^{-1}$

(+ $53 \mathrm{BTU} \cdot 1 \mathrm{~b}^{-1}$ )

$\Delta \mathrm{H}_{\mathrm{g}}$

$+\quad 69 \mathrm{~J} \cdot \mathrm{g}^{-1}$

(+ $30 \mathrm{BTU} \cdot 1 \mathrm{~b}^{-1}$ )

$\Delta H_{v}$

$+297 \mathrm{~J} \cdot \mathrm{g}^{-1}$

$\left(+128 \mathrm{BTU} \cdot 1 \mathrm{~b}^{-1}\right)$

$\Delta H_{\text {ign }}$

- $\quad 4 \mathrm{~J} \cdot \mathrm{g}^{-1}$

(- 2 BTU. $1 \mathrm{~b}^{-1}$ )

$-\Delta H_{s t}$

$16,308 \mathrm{~J} \cdot \mathrm{g}^{-1}$

$\left(7011 \mathrm{BTU} \cdot 1 \mathrm{~b}^{-1}\right)$

imprecision

$\pm 196(1.20 \%)$

$\pm 84$

overall

imprecision $\pm 238(1.46 \%) \quad \pm 102$ 
The results of the experiments on the "unknown" are given in the bottom half of table 10. Definitions are the same except: 1) results are expressed in terms of the sample mass having a moisture content of the unknown experiments; 2) $-\Delta H_{s t}$ for Lot $B$ is calculated from eq. 15 using the mean value of E determined from the calibration experiments; and 3 ) the precision of $-\Delta \mathrm{H}_{\mathrm{st}}$ is calculated using the "Student $t$ " factor for five degrees of freedom at the 95\% confidence level. The overall imprecision in $-\Delta \mathrm{H}_{\mathrm{st}}$ for Lot $\mathrm{B}$ takes into account the imprecision in $E$ from the Lot $A$ experiments. The value of $-\Delta H_{S t}$ for Lot $B$ was $16,308 \mathrm{~J} \cdot \mathrm{g}^{-1}\left(7011 \mathrm{Btu} \cdot 1 \mathrm{~b}^{-1}\right)$. The value of $-\Delta \mathrm{H}_{\mathrm{st}}$ is calculated from bomb calorimetric measurements for Lot B was $16,597 \mathrm{~J} \cdot \mathrm{g}^{-1}\left(7135 \mathrm{Btu} \cdot 1 \mathrm{~b}^{-1}\right)$.

The flow and bomb calorimeter results for measurements on Lot $A$ and Lot $B$ are summarized in Table 11.

Table 11. Comparison of Flow and Bomb Results

$\begin{array}{lll} & \text { Flow } & \text { Bomb } \\ \text { Imprecision, Lot A Measurements } & 0.91 \% & 0.85 \% \\ \text { Imprecision, Lot B Measurements } & 1.20 \%(\mathrm{a}) & 0.46 \% \\ r=\left(\Delta \mathrm{H}_{\mathrm{st}}\right)_{B} /\left(\Delta \mathrm{H}_{\mathrm{st}}\right)_{\mathrm{A}} & 1.009 & 1.027 \\ \text { Uncertainty in } r & 0.027(\mathrm{~b}) & 0.010\end{array}$

(a) Excludes imprecision in $E$.

(b) Sum of imprecision and the systematic error due to moisture content of samples. 
The imprecision of the flow measurements on lot $A$ and lot $B$ were $0.91 \%$ and $1.21 \%$ respectively. (i.e., imprecision of $E$ and $-\Delta H_{s t}$ excluding that due to $E$, respectively). The corresponding imprecision in the calorific values determined with the bomb calorimeter for four samples of lot A was $0.85 \%$ and for four samples of lot B was $0.46 \%$. The ratio, $r$, of $\Delta H_{s t}$ of lot $B$ divided by that of lot $A$ for the flow measurements is 1.009 with an imprecision of 0.015 (i.e. $1.5 \%$ ) at the $95 \%$ confidence level. For purposes of comparison with bomb results, the uncertainty of $r$ is estimated to be \pm 0.027 . This was computed as the sum of the overall imprecision in $r$ plus twice the uncertainty in the mass of the sample ( 0.5 mass percent) due to the uncertainty in moisture content of the RDF-4 samples used in the flow work. The corresponding value of $r$ based on the bomb calorimeter data was 1.027 with an imprecision and uncertainty of \pm 0.010 . The flow and bomb measurements agree within their combined uncertainties.

The average corrections for vaporization of water in table 10 are large because of the very large corrections made in experiments $10 A$ and $11 B$, in which the inlet oxygen was dry (rather than saturated with water). The average correction for the two dry inlet oxygen experiments was $4.3 \%$ of $-\Delta \mathrm{H}_{\text {st }}$. When the inlet oxygen was asturated with water the average correction for vaporization was $1.6 \%$ for Lot $A$ and $1.3 \%$ for $\operatorname{lot} B$. The sum of the average corrections for $\mathrm{CO}$, heat transport by gas flow, and sample ignition were only $0.7 \%$ for Lot $A$ and $1.2 \%$ for Lot $B$.

The average corrections for the heat leak applied to the observed temperature rise were $1.3 \%$ and $2.1 \%$ of $\Delta R$ for $10 t A$ and lot $B$, respectively. The corrections ranged from -0.7 to $+4 \%$ of $\Delta R$. The corrections assume that the temperature of the calorimeter environment is held constant and that steady state heat transfer between the calorimeter water and the environment is achieved rapidly. Since these conditions were not met by the prototype calorimeter, we felt the largest error in the flow measurements would be in the correction for the heat leak. Appropriate experiments plus an error analysis were carried out to determine if this was true. 
An error analysis of the results (see section 4) shows that the total estimated systematic error of the enthalpy ratio, $r$, is between $-2.0 \%$ and $+0.0 \%$ and that the estimated systematic error in $r$ associated with the prototype calorimeter is about $-0.3 \%$. Hence, we expect use of the completed final calorimeter rather than the prototype calorimetric assembly should reduce the systematic error in $r$ to between $-1.7 \%$ and $+0.3 \%$. If the final calorimeter were to be calibrated with cellulose or with an electrical heater, the systematic error would be about the same. A further reduction in the systematic error can only be accomplished in the final calorimeter by changes in the procedures and/or auxiliary measurements used in this preliminary work and further changes in the equipment. These changes will affect future measurements in the final 25 gram capacity flow calorimeter. A summary of the proposed changes and the basis for the proposals is as follows.

1) Heat leak corrections were made assuming no water is vaporized from the calorimeter during the final drift period when the oxygen entering the calorimeter is saturated with water vapor. The estimated maximum correction in $r$ due to the error in this assumption is $-0.7 \%$. The error can be eliminated by allowing no gases to enter or leave the calorimeter during both drift periods.

2) The method used to calculate the correction for water vaporized from the calorimeter during the main period was indirect. It was calculated from the average elemental hydrogen content of each lot of RDF-4 and the amount of water condensed in the water trap in each experiment. A more accurate method is to determine the amount of water vaporized from the calorimeter directly. The accuracy and precision of such a measurement will be greater if the oxygen supplied to the calorimeter is dry. In order to reduce the magnitude of the vaporization correction under this condition, a more efficient water trap is needed and has been constructed. 
3) The results were calculated assuming that all sulfur was oxidized to $\mathrm{SO}_{2}(g)$ and all chlorine converted to hydrochloric acid. All the acid was assumed to be retained in the water trap. Comparison of the measured and predicted acidity of the water condensed in the water trap (i.e. from the elemental chlorine content of each RDF-4 lot) confirmed this assumption. Measurement of the acidity of the water in the product gas scrubber indicated aqueous acid was leaving the calorimeter. Hopefully, this loss can be reduced by the changes made to the water trap to improve its condensation efficiency. The chloride and sulfur content as well as the total acid content of the water trap must be determined in each experiment.

4) The estimated systematic error in $r$ of $\pm 0.5 \%$ due to possible error in the moisture content in each lot of RDF-4 should be reduced by changes in procedure.

5) The reduction in the mass of the ash produced by heating the ash plus crucible and plate with the flame of an oxygen-gas torch corresponded, on the average, to $0.07 \%$ of the original RDF-4 sample mass. The estimated systematic error in $r$ is negligible unless the calorimeter is calibrated with cellulose. In the latter case the error is beween $+0.09 \%$ and $+0.14 \%$. We feel the more probable value is $0.09 \%$. However, the species vaporized in the procedure were not identified. A possible method of eliminating the error is to expose the ash, sample plate, and crucible to a methane-oxygen flame during the last stages of combustion.

We found that the amount of ash formed in the combustions in the flow calorimeter agreed with that determined in the bomb calorimetric measurements and by the ASTM method within their combined experimental imprecision $( \pm 3.5 \%$ for the flow work and $\pm 2 \%$ for the bomb and ASTM work). (Measurement of ash contents for each lot before and after homogenization in a vee-blender suggested some segregation within Lot B may have occurred, but not Lot A. Agreement between the 
amount of ash in the bomb and flow experiments observation is a necessary but not sufficient condition that the enthalpy of reaction in a combustion bomb and flow calorimeter are the same. A subsequent series of measurements in the final flow calorimeter will be needed to relate the bomb and flow measurements .

\section{References}

[1] Kirklin, D.R., Domalski, E.S., and Mitche11, D.J., NBSIR 80-1968.

[2] Prosen, E.J., Maron, F.W., and Rossini, F.D., J. Res. Nat. Bur. Std. 42 , 269 (1949).

[3] Goldberg, R.N., Nutta11, R.L., Prosen, E.J., and Brunetti, A.P. Digital Data Acquisition and Computer Computation Applied to Calorimetric Experiments. Nat. Bur. Stand. (U.S.) Report 10-437; 1971 June.

[4] Wagman, D.D., Evans, W.H., Parker, V.B., Halow, I., Bailey, S.M., and Schumm, R.H., Selected Values of Chemical Thermodynamic Properties. Nat. Bur. Stand. (U.S.) Tech. Note 270-3; 1968 January. 264 p. 


\section{Acknowledgements}

The authors wish to acknowledge the very substantial contributions made by others at NBS to this work: W. Dorko and R. Burke (and colleagues) of the Center for Analytical Chemistry for mass spectrometric and elemental analyses, respectively, of combustion products; S. Abramowitz of the Center for Chemical Physics for infrared spectroscopic analysis of the combustion product gases and for guidance along with $W$. Dorko in selection of instrumentation for analysis of product gases; J. Berke, of the Office of Recycled Materials, for photographing combustions in the combustor development experiments; R.V. Kelly of the Center for Chemical Physics who determined ash and moisture contents of the RDF-4 samples after the flow combustion runs; G.T. Armstrong, D. Garvin, and E.J. Prosen of the Chemical Thermodynamics Division who provided key ideas to the 25 gram combustor development; and E.J. Prosen for advice on flow calorimetry.

This work was jointly sponsored by the NBS Office of Recycled Materials and the U.S. Department of Energy, Office of Energy from Municipal Waste. 
BIBLIOGRAPHIC DATA

NBSIR $22-2457$

SHEET (See instructions)

4. TITLE AND SUBTITLE

\section{Gram Capacity Combustion Flow Calorimeter}

5. AUTHOR(S)

E.S. Domalski, K.L. Churney, M.L. Reilly, D.R. Kirklin, A.E. Ledford, and D.D. Thornton 6. PERFORMING ORGANIZATION (If joint or other than NBS, see instructions)

NATIONAL BUREAU OF STANDARDS

DEPARTMENT OF COMMERCE

WASHINGTON, D.C. 20234

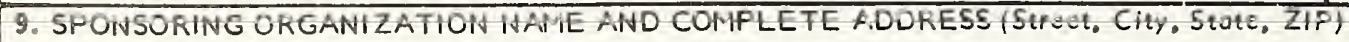

7. Contract/Grant No.

EA-77-A-01-6010

8. Type of Report \& Period Covered INTERIM

1 Oct $1979-30$ Sep 1980

U.S. Department of Energy, Office of Energy from Municipal Waste,

-Forrestal Building, 9000 Independence Avenue, S.W.

Washington, D.C. 20585

10. SUPPLEMENTARY NOTES

Funding equally shared by the NBS Office of Recycled Materials and the

DoE Office of Energy from Municipal Waste.

$\square$ Document describes a computer program; SF-185, FIPS Software Summary, is attached.

11. ABSTRACT (A 200-word or less factual summary of most significant information. If document includes a significant tibliography or literature survey, mention it here)

A new calorimeter is being developed at the National Bureau of Standards to determine the enthalpies of combustion of kilogram-size samples of municipal solid waste (MSW) in flowing oxygen near atmospheric pressure. The organic fraction of 25 gram pellets of highly processed MSW has been burned in pure oxygen to $\mathrm{CO}_{2}$ and $\mathrm{H}_{2} \mathrm{O}$ in a small prototype flow calorimeter. The carbon content of the ash and the uncertainty in the amount of $\mathrm{CO}$ in the combustion products contribute calorimetric errors of 0.1 percent or less to the enthalpy of combustion.

12. KEY WORDS (Six to twelve entries; alphabetical order: capitalize only proper names; and separate key words by semicolons) enthalpy of combustion; flow calorimetry; 25 gram capacity flow calorimeter; municipal solid waste; refuse; refuse-derived-fuel.

14. NO. OF PRINTED PAGES

X Order From National Technical Information Service (NTIS), Springfield, VA. 2216I

$\begin{array}{r}48 \\ \text { PRINTED PAGES } \\ \hline 15 . \text { Price } \\ \$ 7.50 \\ \hline\end{array}$



